\title{
On a diffuse interface model of tumor growth
}

\author{
Sergio Frigeri * $\quad$ Maurizio Grasselli ${ }^{\dagger} \quad$ Elisabetta Rocca ${ }^{\ddagger}$
}

\begin{abstract}
We consider a diffuse interface model of tumor growth proposed by A. HawkinsDaruud et al. This model consists of the Cahn-Hilliard equation for the tumor cell fraction $\varphi$ nonlinearly coupled with a reaction-diffusion equation for $\psi$, which represents the nutrient-rich extracellular water volume fraction. The coupling is expressed through a suitable proliferation function $p(\varphi)$ multiplied by the differences of the chemical potentials for $\varphi$ and $\psi$. The system is equipped with no-flux boundary conditions which entails the conservation of the total mass, that is, the spatial average of $\varphi+\psi$. Here we prove the existence of a weak solution to the associated Cauchy problem, provided that the potential $F$ and $p$ satisfy sufficiently general conditions. Then we show that the weak solution is unique and continuously depends on the initial data, provided that $p$ satisfies slightly stronger growth restrictions. Also, we demonstrate the existence of a strong solution and that any weak solution regularizes in finite time. Finally, we prove the existence of the global attractor in a phase space characterized by an a priori bounded energy.
\end{abstract}

Keywords: diffuse interface, tumor growth, Cahn-Hilliard equations, reactiondiffusion equations, weak solutions, well-posedness, global attractors.

MSC 2010: 35D30; 35K57; 35Q92; 37L30; 92C17.

*Weierstrass Institute for Applied Analysis and Stochastics, Mohrenstr. 39, D-10117 Berlin, Germany. E-mail: SergioPietro.Frigeri@wias-berlin.de The author is supported by the FP7-IDEAS-ERC-StG Grant \#256872 (EntroPhase)

${ }^{\dagger}$ Dipartimento di Matematica, Politecnico di Milano, Milano I-20133, Italy. E-mail: maurizio.grasselli@polimi.it

${ }^{\ddagger}$ Weierstrass Institute for Applied Analysis and Stochastics, Mohrenstr. 39, D-10117 Berlin, Germany. E-mail: Elisabetta.Rocca@wias-berlin.de and Dipartimento di Matematica "F. Enriques", Università degli Studi di Milano, Milano I-20133, Italy. E-mail: elisabetta.rocca@unimi.it The author is supported by the FP7-IDEAS-ERC-StG Grant \#256872 (EntroPhase) 


\section{Introduction}

Modeling tumor growth dynamic has recently become a major issue in applied mathematics (see, for instance, [9, 19], cf. also [2, 24]). The models can be divided into two broad categories: continuum models and discrete or cellular automata models (however, see, e.g., [9, Chap.7] for hybrid continuum-discrete models). Concerning the former ones, the necessity of dealing with multiple interacting constituents has led to consider diffuseinterface models based on continuum mixture theory (see, for instance, [8, 23, 28] and references therein, cf. also [5, 10, 17]). Such models generally consist of Cahn-Hilliard equations with transport and reaction terms which govern various types of cell concentrations. The reaction terms depend on the nutrient concentration (e.g., oxygen) which obeys to an advection-reaction-diffusion equation coupled with the Cahn-Hilliard equations. The cell velocities satisfy a generalized Darcy's (or Brinkman's) law where, besides the pressure gradient, there is also the so-called Korteweg force due to the cell concentration. Numerical simulations of diffuse-interface model for tumor growth have been carried out in several papers (see, for instance, [9, Chap.8] and references therein). Nonetheless, a rigorous mathematical analysis of the resulting systems of differential equations is still in its infancy. In particular, to the best of our knowledge, the first related papers are concerned with the so-called Cahn-Hilliard-Hele-Shaw system (see [20], cf. also [4, 26, 27]) in which the nutrient is neglected. Moreover, a very recent contribution (see [7]) is devoted to analyzing an approximation of a model recently proposed in [16] (see also [29]). In this model, velocities are set to zero and the state variables are reduced to the tumor cell fraction $\varphi$ and the nutrient-rich extracellular water fraction $\psi$. The corresponding PDE system is given by

$$
\begin{aligned}
& \varphi_{t}=\Delta \mu+p(\varphi)(\psi-\mu) \\
& \mu=-\Delta \varphi+F^{\prime}(\varphi) \\
& \psi_{t}=\Delta \psi-p(\varphi)(\psi-\mu)
\end{aligned}
$$

in $\Omega \times(0, \infty)$, where $\Omega \subset \mathbb{R}^{3}$ is a bounded smooth domain. Here $F$ is the typical double-well associated with the Ginzburg-Landau free-energy functional, while $p$ is a proliferation function which must be nonnegative and may have, for instance, the form $p(s)=p_{0}\left(1-s^{2}\right) \chi_{[-1,1]}(s)$ for $s \in \mathbb{R}, p_{0}>0$. Here $\chi_{[-1,1]}$ represents the indicator function of $[-1,1]$.

System (1.1)-(1.3) is equipped with the no-flux boundary conditions

$$
\partial_{n} \varphi=\partial_{n} \mu=\partial_{n} \psi=0 \quad \text { on } \partial \Omega \times(0, \infty) \text {, }
$$

and initial conditions

$$
\varphi(0)=\varphi_{0}, \quad \psi(0)=\psi_{0} \quad \text { in } \Omega .
$$


In [7] the authors consider a relaxed model in which the chemical potential $\mu$ contains a viscous term $\alpha \varphi_{t}, \alpha>0$ and equation (1.1) has an additional term $\alpha \mu_{t}$ which requires a further initial condition. For this model, existence and uniqueness of a variational solution is proven under very general conditions on $F$, while $p$ is supposed to be globally bounded and Lipschitz continuous. Then, imposing substantial restrictions on $F$ (e.g., polynomial growth of order 4 ), the authors prove the existence of a sequence $\left\{\alpha_{n}\right\}$ and a sequence of solutions which converges to a solution to problem (1.1)-(1.5) as $\alpha_{n}$ goes to 0. Such a solution is more regular and unique provided that $\varphi_{0}$ is smooth enough.

Here we want to analyze problem (1.1)-(1.5) without any regularizing term. More precisely, it is not difficult to check that system (1.1)-(1.3) with (1.4) is characterized by the total energy balance law (see [16, (10)])

$$
\frac{d}{d t} \mathcal{E}(\varphi, \psi)+\|\nabla \mu\|^{2}+\|\nabla \psi\|^{2}+\int_{\Omega} p(\varphi)(\mu-\psi)^{2}=0
$$

where the energy $\mathcal{E}$ is given by

$$
\mathcal{E}(\varphi, \psi):=\frac{1}{2}\|\nabla \varphi\|^{2}+\frac{1}{2}\|\psi\|^{2}+\int_{\Omega} F(\varphi) .
$$

Therefore, it seems natural to find a solution assuming that the initial data have just finite energy. This is our first result, namely, existence of a weak solution of finite energy. The assumptions on $F$ and $p$ are more general than the ones in [7] for the case $\alpha=0$. In particular, in the present contribution $p$ can have a polynomially controlled growth. Concerning $F$, we can take any $C^{2}$ and $\lambda_{1}$-convex potential satisfying $\left|F^{\prime}\right| \leq \lambda_{2} F+\lambda_{3}$ for some nonnegative constants $\lambda_{1}, \lambda_{2}, \lambda_{3}$. For instance, $F(s)=\exp (s)$ or $F$ with arbitrary polynomial growth. Also, with a further restriction on the growth of $p^{\prime}$ and assuming $F$ to have a polynomially controlled growth, we can establish the continuous dependence on the initial data (and so the uniqueness of weak solutions).

The proof is obtained by suitably approximating the potential $F$ with a coercive sublinear potential $F_{m}$ and finding an approximating solution of such a problem through a Faedo-Galerkin scheme. The crucial point then consists in obtaining appropriate a priori estimates to pass to the limit via compactness results with respect to $m$. In particular, a bootstrap argument is used in order to derive the optimal regularity estimate for $\varphi$, which is necessary in order to prove the continuous dependence estimate as well as for the analysis of the global longtime behavior.

Then we prove a regularity result which helps us to investigate the global longtime behavior of the solutions. Concerning this issue, observe that conditions (1.4) imply the conservation of the total mass

$$
\int_{\Omega}(\varphi(t)+\psi(t))=\int_{\Omega}\left(\varphi_{0}+\psi_{0}\right), \quad \forall t \geq 0 .
$$

However, we are not able to obtain independent global bounds for the spatial averages of $\varphi(t)$ and $\psi(t)$. On account of this fact, we can show that (1.1)-(1.4) generates a dynamical 
system taking as phase space a bounded set in the finite energy space with a constraint on the total mass. We can thus prove that such a system has a global attractor.

This is just a preliminary step towards the theoretical analysis of more refined models. For instance, one may include the fluid velocity either given as a datum or satisfying a generalized Darcy's (or Brinkman's) law. Also, one should take a logarithmic potential $F$, which is physically more relevant, and nonconstant (possibly degenerate) mobility in the Cahn-Hilliard equation. On the other hand, the free energy functional may contain a nonlocal spatial interaction in place of the usual term $|\nabla \varphi|^{2}$ giving rise to a convolution operator acting on $\varphi$ in place of $\Delta \varphi$ in (1.2) (see, for instance, [28], cf. also [13, 14]). These are just some examples of challenging extensions of the simplified model expressed by (1.1) $-(1.3)$.

Plan of the paper. In Section 2 we define the notation and we recall a useful inequality. In Section 3 we prove that Problem (1.1)-(1.5) admits a unique weak solution (which continuously depends on the data) under proper assumptions on the nonlinearities $F$ and $p$. In Section 4 we establish a regularity result for Problem (1.1)-(1.5) that holds under the same condition on $p$ which ensures uniqueness. This result turns out to be crucial in order to eventually prove the existence of the global attractor.

\section{Notation and preliminaries}

Let $\Omega$ be a sufficiently regular, bounded domain in $\mathbb{R}^{3}$, let $T>0$ and set $Q=\Omega \times(0, T)$. Then we define $H:=L^{2}(\Omega)$ and $V:=H^{1}(\Omega)$ and denote by $\|\cdot\|,(\cdot, \cdot)$ the norm and the scalar product in $H$, respectively. If $X$ is a (real) Banach space, the notation $\langle\cdot$,$\rangle will be$ used to denote the duality pairing between $X$ and its dual $X^{\prime}$. For every $f \in V^{\prime}, \bar{f}$ will stand for the average of $f$ over $\Omega$, i.e., $\bar{f}:=|\Omega|^{-1}\langle f, 1\rangle$. Here $|\Omega|$ is the Lebesgue measure of $\Omega$.

We also introduce the operator $A:=-\Delta+I$ with homogeneous Neumann boundary condition. It is well known that $A: D(A) \subset H \rightarrow H$ is an unbounded linear operator in $H$ with domain

$$
D(A)=\left\{\varphi \in H^{2}(\Omega): \partial_{\mathbf{n}} \varphi=0 \text { on } \partial \Omega\right\},
$$

and that $A^{-1}: H \rightarrow H$ is a self-adjoint compact operator on $H$. By a classical spectral theorem there exist a sequence of eigenvalues $\lambda_{j}$ with $0<\lambda_{1} \leq \lambda_{2} \leq \cdots$ and $\lambda_{j} \rightarrow \infty$, and a family of eigenfunctions $w_{j} \in D(A)$ such that $A w_{j}=\lambda_{j} w_{j}$. The family of $w_{j}$ is an orthonormal basis in $H$ and it is also orthogonal in $V$ and $D(A)$.

We shall repeatedly use the following Gagliardo-Nirenberg inequality in dimension 3 (see, e.g., [3, 11, 12, 22] for more details) 
Lemma 1. Let $1 \leq p_{1}, p_{2} \leq \infty, 0 \leq r<l(r, l \in \mathbb{N})$ and assume that

$$
\theta:=\frac{3 / m-3 / p_{1}-r}{3 / p_{2}-3 / p_{1}-l} \in[r / l, 1)
$$

Then

$$
\|u\|_{W^{r, m}(\Omega)} \leq c\|u\|_{L^{p_{1}(\Omega)}}^{1-\theta}\|u\|_{W^{l, p_{2}(\Omega)}}^{\theta}, \quad \forall u \in W^{l, p_{2}} \cap L^{p_{1}}(\Omega) .
$$

\section{Existence and uniqueness of weak solutions}

In this section we prove that Problem (1.1)-(1.5) admits a weak solution, provided that $F$ and $p$ have polynomial growth with given orders $\rho$ and $q$, respectively. The upper bounds on $\rho$ and $q$ in Theorem 1 ensure the existence of a weak solution with optimal regularity for $\varphi$, i.e., $\varphi \in L^{2}\left(0, T ; H^{3}(\Omega)\right)$. Such assumptions can be relaxed if only existence of the weak solution is required (cf. Corollary 1). An additional restriction on the proliferation function $p$ allows us to prove uniqueness as well as a continuous dependence estimate on the initial data for weak solutions. In any case, our assumptions on $F$ and $p$ are more general than those made in [16] (cf. also [7] when $\alpha=0$ ).

Let us begin with the existence result, which will be proven, for the case where the growth $\rho$ of $F$ is greater than 4 , by means of a double approximation procedure, namely by first exploiting the Faedo-Galerkin scheme to prove existence for $\rho=4$ and then by approximating $F$ with a sequence of potentials having growth which is at most 4 .

The assumptions we need for the existence are the following

(F) $F \in C^{2}(\mathbb{R})$ satisfies

$$
\begin{aligned}
& \left|F^{\prime \prime}(s)\right| \leq c_{1}\left(1+|s|^{\rho-2}\right), \\
& F^{\prime \prime}(s) \geq c_{2}|s|^{\rho-2}-c_{3},
\end{aligned}
$$

for all $s \in \mathbb{R}$, with $c_{1}, c_{2}, c_{3} \geq 0$ and with $\rho \in[4,17 / 3)$.

(P) $p \in C_{l o c}^{0,1}(\mathbb{R})$ satisfies

$$
0 \leq p(s) \leq c_{4}\left(1+|s|^{q}\right)
$$

for all $s \in \mathbb{R}$, with $c_{4}>0$ and with $q \in[1,9)$.

Before stating the existence result, let us introduce the definition of weak solution to Problem (1.1)-(1.5).

Definition 1. Let $\varphi_{0} \in V, \psi_{0} \in H$ and $0<T<\infty$ be given. Then, a pair $[\varphi, \psi]$ is a weak solution to (1.1) -(1.5) on $[0, T]$ if

$$
\varphi \in L^{\infty}(0, T ; V) \cap L^{2}\left(0, T ; H^{2}(\Omega)\right), \quad \varphi_{t} \in L^{r}\left(0, T ; D\left(A^{-1}\right)\right),
$$




$$
\begin{aligned}
& \mu:=-\Delta \varphi+F^{\prime}(\varphi) \in L^{2}(0, T ; V), \\
& \psi \in L^{\infty}(0, T ; H) \cap L^{2}(0, T ; V), \quad \psi_{t} \in L^{r}\left(0, T ; D\left(A^{-1}\right)\right),
\end{aligned}
$$

for some $r>1$, and the following identities are satisfied

$$
\begin{aligned}
& \left\langle\varphi_{t}, \chi\right\rangle+(\nabla \mu, \nabla \chi)=(p(\varphi)(\psi-\mu), \chi), \\
& \left\langle\psi_{t}, \xi\right\rangle+(\nabla \psi, \nabla \xi)=-(p(\varphi)(\psi-\mu), \xi),
\end{aligned}
$$

for all $\chi, \xi \in D(A)$ and for almost all $t \in(0, T)$, together with the initial conditions (1.5).

Remark 1. Notice that the regularity properties of weak solution imply that

$$
\varphi \in C_{w}([0, T] ; V), \quad \psi \in C_{w}([0, T] ; H) .
$$

Hence, the initial conditions (1.5) make sense.

Theorem 1. Assume that $(F)$ and $(P)$ are satisfied. Let $\varphi_{0} \in V$ and $\psi_{0} \in H$. Then, for every $T>0$, Problem (1.1) -(1.5) admits a weak solution on $[0, T]$ such that

$$
\begin{aligned}
& \varphi \in L^{2}\left(0, T ; H^{3}(\Omega)\right), \\
& F(\varphi) \in L^{\infty}\left(0, T ; L^{1}(\Omega)\right), \quad \sqrt{p(\varphi)}(\mu-\psi) \in L^{2}(0, T ; H),
\end{aligned}
$$

which satisfies the following energy inequality

$$
\mathcal{E}(\varphi, \psi)+\int_{0}^{t}\left(\|\nabla \mu\|^{2}+\|\nabla \psi\|^{2}\right) d \tau+\int_{0}^{t} \int_{\Omega} p(\varphi)(\mu-\psi)^{2} \leq \mathcal{E}\left(\varphi_{0}, \psi_{0}\right), \quad \forall t>0
$$

where $\mathcal{E}$ is given by (1.7). Furthermore, if $q \leq 4$, then we have

$$
\varphi_{t}, \psi_{t} \in L^{2}\left(0, T ; V^{\prime}\right)
$$

and (3.11) holds with the equal sign. Moreover, in this case the weak formulation (3.7), (3.8) is satisfied also for all $\chi, \xi \in V$.

The following lemma will turn to be useful in the proof of Theorem 1 (cf. Step II). Indeed, it allows to suitably approximate a regular potential having general $\rho$-growth (in particular in case $\rho>4$ ) and satisfying conditions (3.1), (3.2) with a sequence of regular potentials having sublinear growth.

Lemma 2. Assume that $F$ satisfies (3.1) and (3.2) for some given $\rho \geq 2$. Then, there exists a sequence of $F_{m} \in C^{2}(\mathbb{R})$ with each $F_{m}$ having sublinear growth, such that $F_{m}(s) \rightarrow$ $F(s)$ pointwise for all $s \in \mathbb{R}$ and satisfying, for every $m \in \mathbb{N}$, the bounds

$$
\left|F_{m}(s)\right| \leq|F(s)|, \quad\left|F_{m}^{\prime}(s)\right| \leq k_{1}\left|F^{\prime}(s)\right|, \quad\left|F_{m}^{\prime \prime}(s)\right| \leq k_{2}\left|F^{\prime \prime}(s)\right|, \quad \forall s \in \mathbb{R},
$$


and the equi-coercivity condition

$$
F_{m}(s) \geq k_{3}|s|-k_{4}, \quad \forall s \in \mathbb{R}
$$

where $k_{i}, i=1, \cdots, 4$, are some positive constants which do not depend on $m$ (they depend on $F$ and $\rho$ only).

Proof. Let us first choose an auxiliary function $\eta_{\sigma} \in C^{2}([0, \infty))$, where $\sigma>1$ is fixed, such that $\eta_{\sigma}(0)=1, \eta_{\sigma}^{\prime}(0)=\eta_{\sigma}^{\prime \prime}(0)=0,0 \leq \eta_{\sigma}(s) \leq 1$ for all $s \geq 0, \eta_{\sigma}(s)=1 / s^{\rho-1}$ for all $s \geq \sigma$ and satisfying

$$
\max _{s \in[0, \sigma]}\left|\eta_{\sigma}^{\prime}(s)\right| \leq \frac{a_{1}}{\sigma}, \quad \max _{s \in[0, \sigma]}\left|\eta_{\sigma}^{\prime \prime}(s)\right| \leq \frac{a_{2}}{\sigma^{2}}
$$

where $a_{1}, a_{2}$ are positive constants independent of $\sigma$.

A possible construction of a function satisfying these conditions may be given, e.g., by

$$
\eta_{\sigma}(s)= \begin{cases}b_{1} s^{5}+b_{2} s^{4}+b_{3} s^{3}+1 & 0 \leq s \leq \sigma \\ \frac{1}{s^{\rho-1}} & s \geq \sigma,\end{cases}
$$

where $b_{1}, b_{2}, b_{3}$ are determined in such a way that $\eta_{\sigma} \in C^{2}([0, \infty))$.

Then, for every $m \in \mathbb{N}$ define $\theta_{m}$ as the even extension on $\mathbb{R}$ of the following function

$$
\theta_{m}^{+}(s)= \begin{cases}1 & 0 \leq s \leq m \\ \eta_{m}(s-m) & s \geq m\end{cases}
$$

We can then check that $\theta_{m} \in C^{2}(\mathbb{R}), 0 \leq \theta_{m}(s) \leq 1$ for all $s \in \mathbb{R}, \theta_{m}(s) \rightarrow 1$ as $m \rightarrow \infty$ for all $s \in \mathbb{R}$, and $\max _{s \in \mathbb{R}}\left|\theta_{m}^{\prime}(s)\right| \leq a_{1} / m, \max _{s \in \mathbb{R}}\left|\theta_{m}^{\prime \prime}(s)\right| \leq a_{2} / m^{2}$.

Let us now set

$$
F_{m}(s):=F(s) \theta_{m}(s) .
$$

Then, it is easy to see that, thanks to assumption (F), the sequence of $F_{m}$ constructed in this way satisfies all the required conditions, in particular (3.13) and (3.14). Let us check, e.g., (3.13) ${ }_{2}$. Using (3.1) and (3.2) we first have, for $m \leq s \leq 2 m$

$$
\left|F(s) \theta_{m}^{\prime}(s)\right| \leq \frac{a_{1}}{m} C\left(1+|2 m|^{\rho}\right) \leq C\left(1+|s|^{\rho-1}\right) \leq C\left|F^{\prime}(s)\right|,
$$

where $C$ denotes a positive constant which depends on $F$ and $\rho$ only, but is independent on $m$. On the other hand, for $s \geq 2 m$ we have

$$
\left|F(s) \theta_{m}^{\prime}(s)\right| \leq C \frac{1+|s|^{\rho}}{(s-m)^{\rho}} \leq C\left(1+2^{\rho}\right) .
$$

Hence, collecting (3.15) and (3.16) we get

$$
\left|F_{m}^{\prime}(s)\right| \leq\left|F^{\prime}(s)\right|+\left|F(s) \theta_{m}^{\prime}(s)\right| \leq C\left|F^{\prime}(s)\right|, \quad \forall s \in \mathbb{R}, \forall m .
$$


Inequality $(3.13)_{3}$ can be checked in a similar way. Finally, as far as the equi-coercivity condition (3.14) is concerned, noting that from (3.2) we have $F(s) \geq c_{2}^{\prime}|s|^{\rho}-c_{3}^{\prime}$, for all $s \in \mathbb{R}$, then, for $s \geq 2 m$

$$
F_{m}(s) \geq \frac{c_{2}^{\prime}|s|^{\rho}-c_{3}^{\prime}}{(s-m)^{\rho-1}} \geq c_{2}^{\prime}|s|-\frac{c_{3}^{\prime}}{2^{\rho-1}},
$$

and, for $0 \leq s \leq 2 m$ we have simply $F(s) \theta_{m}(s) \geq-c_{3}^{\prime} \theta_{m}(s) \geq-c_{3}^{\prime}$. Hence, we immediately get (3.14).

Proof of Theorem 1]. Step I (case $\rho=4)$.

Let us first prove the existence of a weak solution with optimal regularity (3.9) under the assumption that $F$ has growth 4 at most. We shall use a Faedo-Galerkin approximation method. Let us then take the family $\left\{w_{j}\right\}_{j \geq 1}$ of the eigenfunctions of $A$ as a Galerkin basis in $V$, and let $P_{n}$ be the orthogonal projectors in $H$ onto the $n$-dimensional subspace $\mathcal{W}_{n}:=\left\langle w_{1}, \cdots w_{n}\right\rangle$ spanned by the first $n$ eigenfunctions. For $n \in \mathbb{N}$ fixed, we look for three functions of the form

$$
\varphi_{n}(t):=\sum_{k=1}^{n} a_{k}^{n}(t) w_{k}, \quad \psi_{n}(t):=\sum_{k=1}^{n} b_{k}^{n}(t) w_{k}, \quad \mu_{n}(t):=\sum_{k=1}^{n} c_{k}^{n}(t) w_{k}
$$

that solve the following approximating problem

$$
\begin{aligned}
& \left(\varphi_{n}^{\prime}, w_{j}\right)+\left(\nabla \mu_{n}, \nabla w_{j}\right)=\left(p\left(\varphi_{n}\right)\left(\psi_{n}-\mu_{n}\right), w_{j}\right), \\
& \left(\mu_{n}, w_{j}\right)=\left(\nabla \varphi_{n}, \nabla w_{j}\right)+\left(F^{\prime}\left(\varphi_{n}\right), w_{j}\right), \\
& \left(\psi_{n}^{\prime}, w_{j}\right)+\left(\nabla \psi_{n}, \nabla w_{j}\right)=-\left(p\left(\varphi_{n}\right)\left(\psi_{n}-\mu_{n}\right), w_{j}\right), \\
& \varphi_{n}(0)=\varphi_{0 n}, \quad \psi_{n}(0)=\psi_{0 n},
\end{aligned}
$$

for $j=1, \cdots, n$, where $\varphi_{0 n}:=P_{n} \varphi_{0}$ and $\psi_{0 n}:=P_{n} \psi_{0}$ (prime denote the derivative with respect to time).

It is easy to see that solving the approximate problem (3.17)-(3.20) is equivalent to solving a Cauchy problem for a system of $2 n$ ordinary differential equations in the $2 n$ unknowns $a_{j}^{n}, b_{j}^{n}$. Since $F^{\prime} \in C^{1}$ and $p \in C_{l o c}^{0,1}$, the Cauchy-Lipschitz theorem ensures that there exists $T_{n}^{*} \in(0, \infty]$ such that this system has a unique maximal solution $\mathbf{a}^{n}:=$ $\left(a_{1}^{n}, \cdots, a_{n}^{n}\right), \mathbf{b}^{n}:=\left(b_{1}^{n}, \cdots, b_{n}^{n}\right)$ on $\left[0, T_{n}^{*}\right)$ with $\mathbf{a}^{n}, \mathbf{b}^{n} \in C^{1}\left(\left[0, T_{n}^{*}\right) ; \mathbb{R}^{n}\right)$. Hence, the approximate problem (3.17) -(3.20) admits a unique solution $\varphi_{n}, \psi_{n}, \mu_{n} \in C^{1}\left(\left[0, T_{n}^{*}\right) ; \mathcal{W}_{n}\right)$.

We now deduce the basic estimates on the sequence of approximating solutions. In particular, these estimates will guarantee that $T_{n}^{*}=\infty$ for every $n \in \mathbb{N}$.

Multiply then (3.17) by $c_{j}^{n}$, (3.18) by $a_{j}^{n \prime}$, (3.19) by $b_{j}^{n}$ and sum the resulting identities over $j=1, \cdots, n$. We get the following energy identity satisfied by the solution of the approximate problem

$$
\frac{d}{d t}\left(\frac{1}{2}\left\|\nabla \varphi_{n}\right\|^{2}+\frac{1}{2}\left\|\psi_{n}\right\|^{2}+\int_{\Omega} F\left(\varphi_{n}\right)\right)+\left\|\nabla \mu_{n}\right\|^{2}+\left\|\nabla \psi_{n}\right\|^{2}+\int_{\Omega} p\left(\varphi_{n}\right)\left(\mu_{n}-\psi_{n}\right)^{2}=0 .
$$


By integrating (3.21) in time between 0 and $t$, using (F), (P) and the assumptions on the initial data we immediately deduce the following estimates

$$
\begin{aligned}
& \left\|\varphi_{n}\right\|_{L^{\infty}(0, T ; V)} \leq C, \quad\left\|\psi_{n}\right\|_{L^{\infty}(0, T ; H) \cap L^{2}(0, T ; V)} \leq C, \\
& \left\|\nabla \mu_{n}\right\|_{L^{2}(0, T ; H)} \leq C, \quad\left\|\sqrt{p\left(\varphi_{n}\right)}\left(\psi_{n}-\mu_{n}\right)\right\|_{L^{2}(0, T ; H)} \leq C, \\
& \left\|F\left(\varphi_{n}\right)\right\|_{L^{\infty}\left(0, T ; L^{1}(\Omega)\right)} \leq C .
\end{aligned}
$$

where henceforth $C=C\left(\left\|\varphi_{0}\right\|_{V},\left\|\psi_{0}\right\|\right)$ denotes a nonnegative constant depending on the norms of the initial data (and on $F, \Omega$ ).

Let us now control the sequence of the averages of $\mu_{n}$. From (3.18) we get

$$
\left|\left(\mu_{n}, 1\right)\right|=\left|\left(F^{\prime}\left(\varphi_{n}\right), 1\right)\right| \leq c_{5}\left(F\left(\varphi_{n}\right), 1\right)+c_{6} \leq C,
$$

where $c_{5}, c_{6}$ are two nonnegative constants depending only on $F, \Omega$ and where we have used assumption (F) and (3.24). Therefore, the sequence of $\bar{\mu}_{n}$ is bounded in $L^{\infty}(0, T)$ and this bound, together with the first of (3.23) yields

$$
\left\|\mu_{n}\right\|_{L^{2}(0, T ; V)} \leq C
$$

We now prove that the sequence of $\varphi_{n}$ is controlled in $L^{\infty}(0, T ; V) \cap L^{2}\left(0, T ; H^{3}(\Omega)\right)$. Indeed, notice first that (3.18) can be written as

$$
\mu_{n}=-\Delta \varphi_{n}+P_{n} F^{\prime}\left(\varphi_{n}\right)
$$

Observe now that $\left\|P_{n} F^{\prime}\left(\varphi_{n}\right)\right\| \leq\left\|F^{\prime}\left(\varphi_{n}\right)\right\|$. Thus, the sequence of $\varphi_{n}$ is bounded in $L^{\infty}\left(0, T ; L^{6}(\Omega)\right)$, we deduce from (3.1) the bound

$$
\left\|F^{\prime}\left(\varphi_{n}\right)\right\|_{L^{\infty}(0, T ; H)} \leq C
$$

Hence, (3.27) and (3.26) entail that the sequence of $-\Delta \varphi_{n}+\varphi_{n}$ is bounded in $L^{2}(0, T ; H)$ and, on account of the homogeneous Neumann boundary condition for $\varphi_{n}$, a classical elliptic regularity result implies

$$
\left\|\varphi_{n}\right\|_{L^{\infty}(0, T ; V) \cap L^{2}\left(0, T ; H^{2}(\Omega)\right)} \leq C .
$$

By using inequality (2.1), we deduce from (3.29) that the sequence of $\varphi_{n}$ is bounded in $L^{10}(Q)$ and moreover the sequence of $\nabla \varphi_{n}$ is bounded in $L^{\infty}(0, T ; H) \cap L^{2}(0, T ; V) \hookrightarrow$ $L^{10 / 3}(Q)$. On the other hand, we have

$$
\left\|\nabla\left(P_{n} F^{\prime}\left(\varphi_{n}\right)\right)\right\| \leq\left\|A^{1 / 2} P_{n} F^{\prime}\left(\varphi_{n}\right)\right\|=\left\|P_{n} A^{1 / 2} F^{\prime}\left(\varphi_{n}\right)\right\| \leq\left\|\nabla F^{\prime}\left(\varphi_{n}\right)\right\|+\left\|F^{\prime}\left(\varphi_{n}\right)\right\|,
$$

and hence (3.1) together with (3.28) and $(3.22)_{1}$ entail

$$
\left\|P_{n} F^{\prime}\left(\varphi_{n}\right)\right\|_{L^{2}(0, T ; V)} \leq\left\|F^{\prime \prime}\left(\varphi_{n}\right) \nabla \varphi_{n}\right\|_{L^{2}(Q)}+\left\|F^{\prime}\left(\varphi_{n}\right)\right\|_{L^{2}(Q)}
$$




$$
\begin{aligned}
& \leq\left\|F^{\prime \prime}\left(\varphi_{n}\right)\right\|_{L^{5}(Q)}\left\|\nabla \varphi_{n}\right\|_{L^{10 / 3}(Q)}+\left\|F^{\prime}\left(\varphi_{n}\right)\right\|_{L^{2}(Q)} \\
& \leq c\left(1+\left\|\varphi_{n}\right\|_{L^{10}(Q)}^{2}\right)\left\|\nabla \varphi_{n}\right\|_{L^{10 / 3}(Q)}+\left\|F^{\prime}\left(\varphi_{n}\right)\right\|_{L^{2}(Q)} \\
& \leq C
\end{aligned}
$$

By comparison in (3.27), using (3.26) and the elliptic regularity result again, we infer

$$
\left\|\varphi_{n}\right\|_{L^{\infty}(0, T ; V) \cap L^{2}\left(0, T ; H^{3}(\Omega)\right)} \leq C .
$$

We now deduce the estimates for the sequences of time derivatives $\varphi_{n}^{\prime}$ and $\psi_{n}^{\prime}$. Take $\chi \in D(A) \hookrightarrow L^{\infty}(\Omega)$ and write it as $\chi=\chi_{1}+\chi_{2}$, where $\chi_{1}=P_{n} \chi \in \mathcal{W}_{n}$ and $\chi_{2} \in$ $\left(I-P_{n}\right) \chi \in \mathcal{W}_{n}^{\perp}$ (recall that $\chi_{1}, \chi_{2}$ are orthogonal in $H, V$ and $D(A)$ ). Then, from (3.17) we have

$$
\left\langle\varphi_{n}^{\prime}, \chi\right\rangle=\left\langle\varphi_{n}^{\prime}, \chi_{1}\right\rangle=-\left(\nabla \mu_{n}, \nabla \chi_{1}\right)+\left(p\left(\varphi_{n}\right)\left(\psi_{n}-\mu_{n}\right), \chi_{1}\right)
$$

and a similar identity follows from (3.19). Observe that

$$
\begin{aligned}
\left|\left(p\left(\varphi_{n}\right)\left(\psi_{n}-\mu_{n}\right), \chi_{1}\right)\right| & \leq\left\|p\left(\varphi_{n}\right)\right\|_{L^{6 / 5}(\Omega)}\left\|\psi_{n}-\mu_{n}\right\|_{L^{6}(\Omega)}\left\|\chi_{1}\right\|_{L^{\infty}(\Omega)} \\
& \leq c\left\|p\left(\varphi_{n}\right)\right\|_{L^{6 / 5}(\Omega)}\left\|\psi_{n}-\mu_{n}\right\|_{L^{6}(\Omega)}\|\chi\|_{D(A)} .
\end{aligned}
$$

The term $\left(\psi_{n}-\mu_{n}\right)$ is controlled in $L^{2}\left(0, T ; L^{6}(\Omega)\right)$, then we need to control the sequence of $p\left(\varphi_{n}\right)$ in $L^{\sigma}\left(0, T ; L^{6 / 5}(\Omega)\right)$ with some $\sigma>2$ in order to get the control of the sequences of $\varphi_{n}^{\prime}, \psi_{n}^{\prime}$ in $L^{r}\left(0, T ; D\left(A^{-1}\right)\right)$ with some $r>1$. From assumption (P) it follows

$$
\left\|p\left(\varphi_{n}\right)\right\|_{L^{\sigma}\left(0, T ; L^{6 / 5}(\Omega)\right)} \leq c\left(1+\left\|\varphi_{n}\right\|_{L^{\sigma q}\left(0, T ; L^{6 q / 5}(\Omega)\right)}^{q}\right) .
$$

But we know that the sequence of $\varphi_{n}$ is bounded in $L^{\infty}(0, T ; V) \cap L^{2}\left(0, T ; H^{3}(\Omega)\right.$ ) (cf. (3.30) $)$, and, thanks to inequality (2.1), we have the following embedding

$$
L^{\infty}(0, T ; V) \cap L^{2}\left(0, T ; H^{3}(\Omega)\right) \hookrightarrow L^{8 \theta /(\theta-6)}\left(0, T ; L^{\theta}(\Omega)\right), \quad \text { for } 6 \leq \theta \leq \infty .
$$

Hence, choosing $\theta=54 / 5$, we obtain

$$
\left\|\varphi_{n}\right\|_{L^{18}\left(0, T ; L^{54 / 5}(\Omega)\right)} \leq C,
$$

and, recalling that $q<9$, we have $L^{18}\left(0, T ; L^{54 / 5}(\Omega)\right) \hookrightarrow L^{\sigma q}\left(0, T ; L^{6 q / 5}(\Omega)\right)$ for some $\sigma>2$. Summing up, we have proven the following bounds

$$
\left\|\varphi_{n}^{\prime}\right\|_{L^{r}\left(0, T ; D\left(A^{-1}\right)\right)} \leq C, \quad\left\|\psi_{n}^{\prime}\right\|_{L^{r}\left(0, T ; D\left(A^{-1}\right)\right)} \leq C, \quad \text { for some } r>1
$$

where we have used $(3.32)$ and $(3.23){ }_{1}$ in (3.31) to get the first bound and $(3.32)$ and $(3.22)_{2}$ to obtain the second bound.

We now deduce from estimates (3.22), (3.26), (3.30) and (3.35) the existence of three functions $\varphi \in L^{\infty}(0, T ; V) \cap L^{2}\left(0, T ; H^{3}(\Omega)\right), \psi \in L^{\infty}(0, T ; H) \cap L^{2}(0, T ; V)$ and 
$\mu \in L^{2}(0, T ; V)$, with $\varphi_{t}, \psi_{t} \in L^{r}\left(0, T ; D\left(A^{-1}\right)\right)$ which are the (weak) limits (up to subsequences) of $\varphi_{n}, \psi_{n}, \mu_{n}$ and $\varphi_{n}^{\prime}, \psi_{n}^{\prime}$, respectively. In order to pass to the limit in the approximate problem, we first observe that thanks to the compact embedding

$$
L^{\infty}(0, T ; V) \cap W^{1, r}\left(0, T ; D\left(A^{-1}\right)\right) \hookrightarrow \hookrightarrow C\left([0, T] ; L^{\kappa}(\Omega)\right), \quad 2 \leq \kappa<6
$$

given by the Aubin-Lions lemma (see, e.g., [18]), we deduce that, up to a subsequence, $\varphi_{n} \rightarrow \varphi$ pointwise almost everywhere in $Q=\Omega \times(0, T)$. Then, since $\left(\psi_{n}-\mu_{n}\right)$ converges weakly to $(\psi-\mu)$ in $L^{2}\left(0, T ; L^{6}(\Omega)\right)$, in order to pass to the limit in $\left(p\left(\varphi_{n}\right)\left(\psi_{n}-\mu_{n}\right), w_{j}\right)$ on the right hand side of (3.17) and (3.19) it is enough that $p\left(\varphi_{n}\right)$ converges strongly to $p(\varphi)$ in $L^{2}\left(0, T ; L^{6 / 5}(\Omega)\right)$. But $\varphi_{n}$ converges to $\varphi$ weakly in $L^{18}\left(0, T ; L^{54 / 5}(\Omega)\right)$. Hence, by also using the pointwise convergence of $\varphi_{n}$ to $\varphi$, we get

$$
\varphi_{n} \rightarrow \varphi, \quad \text { strongly in } L^{\alpha}\left(0, T ; L^{\beta}(\Omega)\right), \quad 2 \leq \alpha<18, \quad 2 \leq \beta<54 / 5 .
$$

Thus, on account of (3.32) (written with $\sigma=2$ ) and of assumption $q<9$, by means of the generalized Lebesgue theorem we infer from (3.36) that

$$
p\left(\varphi_{n}\right) \rightarrow p(\varphi), \quad \text { strongly in } L^{2}\left(0, T ; L^{6 / 5}(\Omega)\right) .
$$

This convergence, combined with the weak convergence $\left(\mu_{n}-\psi_{n}\right) \rightarrow(\mu-\psi)$ in $L^{2}\left(0, T ; L^{6}(\Omega)\right)$, allows us to pass to the limit in the nonlinear term on the right hand side of (3.17) and (3.19) (recall that $w_{j} \in C^{\infty}(\bar{\Omega})$ ). By means of the convergences deduced above we can therefore pass to the limit in the approximate problem (3.17)-(3.20) and deduce that $\varphi, \psi, \mu$ satisfy (3.7)-(3.8). The argument is standard and the details are left to the reader.

The energy inequality (3.11) can be proven by integrating in time (3.21) between 0 and $t$ and passing to the limit as $n \rightarrow \infty$ in the resulting identity. The only nontrivial point is the following inequality

$$
\int_{0}^{t} \int_{\Omega} p(\varphi)(\mu-\psi)^{2} \leq \liminf _{n \rightarrow \infty} \int_{0}^{t} \int_{\Omega} p\left(\varphi_{n}\right)\left(\mu_{n}-\psi_{n}\right)^{2}
$$

We know from (3.33) written for $\theta=14$, that the sequence of $\varphi_{n}$ is bounded in $L^{14}(Q)$ and hence, on account of $(\mathrm{P})$, the sequence of $\sqrt{p\left(\varphi_{n}\right)}$ is bounded in $L^{28 / q}(Q)$. Since $\varphi_{n} \rightarrow \varphi$ also pointwise a.e. in $Q$, then we have $\sqrt{p\left(\varphi_{n}\right)} \rightarrow \sqrt{p(\varphi)}$ strongly in $L^{\gamma}(Q)$, for every $\gamma<28 / q$. In particular we have $\sqrt{p\left(\varphi_{n}\right)} \rightarrow \sqrt{p(\varphi)}$ strongly in $L^{3}(Q)$. Therefore, we have

$$
\sqrt{p\left(\varphi_{n}\right)}\left(\mu_{n}-\psi_{n}\right) \rightarrow \sqrt{p(\varphi)}(\mu-\psi), \quad \text { in } L^{6 / 5}(Q)
$$

and, due $(\underline{3.23})_{2}$, this last weak convergence is also in $L^{2}(Q)$. Hence, (3.38) follows. 
Moreover, if $q \leq 4$ we can easily deduce the regularity $\varphi_{t}, \psi_{t} \in L^{2}\left(0, T ; V^{\prime}\right)$ by comparison in the variational formulation of (1.1) and (1.3). Indeed, estimating the term $p(\varphi)(\psi-\mu)$ in $V^{\prime}$, we get

$$
\|p(\varphi)(\psi-\mu)\|_{V^{\prime}} \leq c\|p(\varphi)\|_{L^{3 / 2}(\Omega)}\|\psi-\mu\|_{L^{6}(\Omega)} .
$$

But, since $q \leq 4$ and $\varphi \in L^{\infty}\left(0, T ; L^{6}(\Omega)\right)$, then assumption (P) implies that we have $p(\varphi) \in L^{\infty}\left(0, T ; L^{3 / 2}(\Omega)\right)$ and therefore, on account of (3.5) and of (3.6) $)_{1}$, (3.39) entails

$$
p(\varphi)(\psi-\mu) \in L^{2}\left(0, T ; V^{\prime}\right) .
$$

Hence, (3.12) follows immediately.

Finally, by choosing $\mu$ and $\psi$ as test functions in the variational formulation of (1.1) and (1.3) (with test functions now in $V$ ), respectively, and using the regularity for $\varphi_{t}, \psi_{t}$ and the chain rule applied to the product $\left\langle\varphi_{t}, F^{\prime}(\varphi)\right\rangle$ (see [6, Proposition 4.2]; notice that (3.2) ensures that $F$ is a quadratic perturbation of a convex function), we obtain

$$
\frac{d}{d t}\left(\frac{1}{2}\|\nabla \varphi\|^{2}+\frac{1}{2}\|\psi\|^{2}+\int_{\Omega} F(\varphi)\right)+\|\nabla \mu\|^{2}+\|\nabla \psi\|^{2}+\int_{\Omega} p(\varphi)(\mu-\psi)^{2}=0 .
$$

By integrating the energy identity (3.40) in time between 0 and $t$ we deduce (3.11) with the equal sign for all $t>0$. This completes the proof of the theorem for the case $\rho=4$.

Step II (case $4<\rho<17 / 3$ ).

In this case we first approximate the potential $F$ with a sequence of potentials $F_{m} \in$ $C^{2}(\mathbb{R})$ satisfying the conditions stated in Lemma 2 ,

Let us now consider problem (1.1) $-(1.5)$ with $F$ replaced by $F_{m}$ and call it Problem $\mathrm{P}_{m}$. Since $F_{m}$ satisfies condition $(\mathrm{F})$ with $\rho \leq 4\left(F_{m}\right.$ has sublinear growth on $\left.\mathbb{R}\right)$ then, for each $m \in \mathbb{N}$, Step I ensures the existence of a weak solution $\left[\varphi_{m}, \psi_{m}\right]$ to Problem $\mathrm{P}_{m}$ such that $\varphi_{m} \in L^{\infty}(0, T ; V) \cap L^{2}\left(0, T ; H^{3}(\Omega)\right), \psi_{m} \in L^{\infty}(0, T ; H) \cap L^{2}(0, T ; V), \mu_{m} \in L^{2}(0, T ; V)$ and satisfying the energy inequality (3.11).

Due to (3.11) (written for each solution $\varphi_{m}, \psi_{m}$ with $F_{m}$ in place of $F$ ), assumptions $(\mathrm{F})$ and $(\mathrm{P}),(3.13)_{1}$ and (3.14), we can argue as for the Faedo-Galerkin approximating solutions $\left[\varphi_{n}, \psi_{n}\right]$ (cf. Step I) and we can still recover the basic estimates (3.22), (3.26) for the sequences of $\varphi_{m}$ and $\psi_{m}$ (notice that in Problem $\mathrm{P}_{m}$ the initial conditions are not approximated).

We now show that the sequence $\varphi_{m}$ is still controlled in $L^{\infty}(0, T ; V) \cap L^{2}\left(0, T ; H^{3}(\Omega)\right)$. This bound will be achieved through an iteration argument.

Notice first that the fact that the sequence of $\varphi_{m}$ is bounded in $L^{\infty}\left(0, T ; L^{6}(\Omega)\right)$ and (3.1) together with the second of (3.13) imply that the sequence of $F_{m}^{\prime}\left(\varphi_{m}\right)$ is bounded in $L^{\infty}\left(0, T ; L^{6 /(\rho-1)}(\Omega)\right)$. Hence, from (1.2) and (3.26) we infer that the sequence of $-\Delta \varphi_{m}+\varphi_{m}$ is bounded in $L^{2}\left(0, T ; L^{6 /(\rho-1)}(\Omega)\right)$, and due to the homogeneous Neumann 
boundary condition for $\varphi_{m}$, by using elliptic regularity theory (see, e.g., [1, 15, 21]) we get

$$
\left\|\varphi_{m}\right\|_{L^{\infty}(0, T ; V) \cap L^{2}\left(0, T ; W^{2, \frac{6}{\rho-1}}(\Omega)\right)} \leq C .
$$

Thanks to inequality (2.1), we deduce from (3.41) that the sequence of $\varphi_{m}$ is bounded in $L^{2(9-\rho)}(Q)$. Moreover, $\nabla \varphi_{m}$ is bounded in $L^{\infty}(0, T ; H) \cap L^{2}\left(0, T ; W^{1,6 /(\rho-1)}(\Omega)\right) \hookrightarrow$ $L^{2(9-\rho) / 3}(Q)$. Therefore, using $(3.13)_{3}$ and (3.1) we get

$$
\begin{aligned}
\left\|\nabla F_{m}^{\prime}\left(\varphi_{m}\right)\right\|_{L^{s_{0}}(Q)} & \leq k_{2}\left\|F^{\prime \prime}\left(\varphi_{m}\right) \nabla \varphi_{m}\right\|_{L^{s_{0}}(Q)} \\
& \leq k_{2}\left\|F^{\prime \prime}\left(\varphi_{m}\right)\right\|_{L^{2(9-\rho) /(\rho-2)}(Q)}\left\|\nabla \varphi_{m}\right\|_{L^{2(9-\rho) / 3}(Q)} \\
& \leq c\left(1+\left\|\varphi_{m}\right\|_{L^{2(9-\rho)}(Q)}^{\rho-2}\left\|\nabla \varphi_{m}\right\|_{L^{2(9-\rho) / 3}(Q)}\right. \\
& \leq C, \quad s_{0}=\frac{2(9-\rho)}{\rho+1} .
\end{aligned}
$$

Notice that $s_{0} \in(1,2]$ since $\rho \in[4,17 / 3)$. In addition, owing to (3.13) $)_{2}$, (3.1) and (3.41), the sequence of $F_{m}^{\prime}\left(\varphi_{m}\right)$ is bounded in $L^{\infty}\left(0, T ; L^{6 /(\rho-1)}(\Omega)\right)$ and hence also in $L^{\infty}\left(0, T ; L^{s_{0}}(\Omega)\right)\left(\right.$ since $\left.6 /(\rho-1)>s_{0}\right)$. Thus we obtain

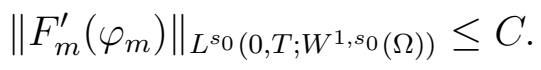

By comparison in (1.2) and using (3.26) and elliptic regularity again, we deduce

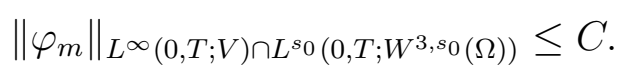

We can now repeat the argument above and improve the estimates for the sequence of $\varphi_{m}$ by means of a bootstrap procedure performed for a finite number of steps. Indeed, observe first that, thanks to (2.1), we have (for any $s \in(1,2]$ )

$$
\begin{aligned}
& \mathbb{X}_{s}:=L^{\infty}(0, T ; V) \cap L^{s}\left(0, T ; W^{3, s}(\Omega)\right) \hookrightarrow L^{7 s}(Q), \\
& \mathbb{Y}_{s}:=L^{\infty}(0, T ; H) \cap L^{s}\left(0, T ; W^{2, s}(\Omega)\right) \hookrightarrow L^{\frac{7}{3} s}(Q) .
\end{aligned}
$$

Taking (3.42) - (3.44) into account, the sequences of $\varphi_{m}$ and $\nabla \varphi_{m}$ are bounded in $L^{7 s_{0}}(Q)$ and in $L^{7 s_{0} / 3}(Q)$, respectively. Hence, by means of (3.1) and $(\underline{3.13})_{3}$, we have

$$
\left\|\nabla F_{m}^{\prime}\left(\varphi_{m}\right)\right\|_{L^{7 s_{0} /(\rho+1)}(Q)} \leq k_{2}\left\|F^{\prime \prime}\left(\varphi_{m}\right) \nabla \varphi_{m}\right\|_{L^{7 s_{0} /(\rho+1)}(Q)} \leq C .
$$

On the other hand, we also have (for any $s \in(1,2]$ )

$$
\mathbb{Z}_{s}:=L^{\infty}\left(0, T ; L^{\frac{6}{\rho-1}}(\Omega)\right) \cap L^{s}\left(0, T ; W^{1, s}(\Omega)\right) \hookrightarrow L^{\frac{\rho+1}{\rho-1} s}(Q) \hookrightarrow L^{\frac{7}{\rho+1} s}(Q),
$$

and since $F_{m}^{\prime}\left(\varphi_{m}\right)$ is bounded in $\mathbb{Z}_{s_{0}}$ thanks to (3.42), (3.41), (3.1) and (3.13) ${ }_{2}, F_{m}^{\prime}\left(\varphi_{m}\right)$ is bounded in $L^{7 s_{0} /(\rho+1)}(Q)$. We therefore deduce that

$$
\left\|F_{m}^{\prime}\left(\varphi_{m}\right)\right\|_{L^{s_{1}\left(0, T ; W^{1, s_{1}}(\Omega)\right)}} \leq C, \quad s_{1}:=\frac{7}{\rho+1} s_{0}
$$


If $s_{1} \geq 2$, then by comparison in (1.2) and using (3.26) and elliptic regularity, we get the desired bound for the sequence of $\varphi_{m}$ in $L^{\infty}(0, T ; V) \cap L^{2}\left(0, T ; H^{3}(\Omega)\right)$. If $s_{1}<2$ then, by comparison in (1.2) and using (3.26) and elliptic regularity, we infer

$$
\left\|\varphi_{m}\right\|_{L^{\infty}(0, T ; V) \cap L^{s_{1}}\left(0, T ; W^{3, s_{1}}(\Omega)\right)} \leq C .
$$

Repeating the argument we now have the sequences of $\varphi_{m}$ and $\nabla \varphi_{m}$ bounded in $\mathbb{X}_{s_{1}}$ and in $\mathbb{Y}_{s_{1}}$, respectively, and hence $\left\|\nabla F_{m}^{\prime}\left(\varphi_{m}\right)\right\|_{L^{7 s_{1} /(\rho+1)}(Q)} \leq C$. Moreover, we know that the sequence of $F_{m}^{\prime}\left(\varphi_{m}\right)$ is bounded in $L^{7 s_{1} /(\rho+1)}(Q)$. This implies

$$
\left\|F_{m}^{\prime}\left(\varphi_{m}\right)\right\|_{L^{s_{2}\left(0, T ; W^{\left.1, s_{2}(\Omega)\right)}\right.}} \leq C, \quad s_{2}:=\left(\frac{7}{\rho+1}\right)^{2} s_{0} .
$$

Again, if $s_{2} \geq 2$ we get the desired claim; otherwise, by using elliptic regularity we infer that the sequence of $\varphi_{m}$ is bounded in $\mathbb{X}_{s_{2}}$ and we repeat the previous argument. By iterating the procedure $k$ times (assuming in particular that $s_{0}<2$ ) we get

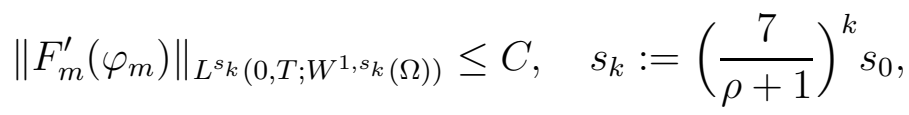

and after a finite number of steps, as soon as we get $s_{k} \geq 2$, the bootstrap procedure ends yielding the bound of the sequence of $\varphi_{m}$ in $L^{\infty}(0, T ; V) \cap L^{2}\left(0, T ; H^{3}(\Omega)\right.$ ) (which cannot be improved since the regularity of $\varphi_{m}$ is related through (1.2) to $\mu_{m} \in L^{2}(0, T ; V)$ ).

As far as the estimates for the sequences of time derivatives $\varphi_{m}^{\prime}, \psi_{m}^{\prime}$ are concerned, the argument is exactly the same as for the sequences of time derivatives $\varphi_{n}^{\prime}, \psi_{n}^{\prime}$ of the Faedo-Galerkin approximating solutions (cf. Step I). Hence, (3.35) still holds for $\varphi_{m}^{\prime}, \psi_{m}^{\prime}$.

Finally, the passage to the limit in Problem $\mathrm{P}_{m}$ (notice that $F_{m}^{\prime}\left(\varphi_{m}\right) \rightarrow F^{\prime}(\varphi)$ pointwise almost everywhere in $Q)$, the proof of the energy inequality (3.11) for $q \in[1,9)$, the proofs of (3.12) and of the energy identity for $q \leq 4$ can be carried out along as done at the end of Step I.

The existence of a weak solution without the the optimal regularity $\varphi \in L^{2}\left(0, T ; H^{3}(\Omega)\right)$ can still be ensured under a more general assumption on $F$, provided we impose a slight restriction (i.e., $q<7$ ) on the growth of $p$.

More precisely, we have the following

Corollary 1. Assume that $F \in C^{2}(\mathbb{R})$ satisfies

$(\mathbf{F})_{1} F^{\prime \prime}(s) \geq-\lambda_{1}$,

$(\mathbf{F})_{2}\left|F^{\prime}(s)\right| \leq \lambda_{2} F(s)+\lambda_{3}$,

for all $s \in \mathbb{R}$, where $\lambda_{1}, \lambda_{2}, \lambda_{3}$ are some nonnegative constants. Moreover, assume that $p \in C_{\text {loc }}^{0,1}(\mathbb{R})$ satisfies (3.3) with $q \in[1,7)$. Let $\varphi_{0} \in V$ and $\psi_{0} \in H$. Then, for every $T>0$ Problem (1.1) -(1.5) admits a weak solution on $[0, T]$ satisfying (3.4) -(3.6), (3.10) and the energy inequality (3.11). Finally, if $q \leq 4$, then we have (3.12) and (3.11) holds with the equal sign. 
Proof. We can follow the Faedo-Galerkin approximation procedure in Step I of the proof of Theorem 1, assuming first that $\varphi_{0} \in D(A)$ in order to control the sequence of $\int_{\Omega} F\left(\varphi_{0 n}\right)$ in the identity obtained by integrating (3.21) in time. Existence of weak solution in the case $\varphi_{0} \in V$ can then be recovered by means of a density argument. The basic estimates (3.22) -(3.24) still hold, as well as the controls (3.25), ensured by $(\mathrm{F})_{2}$, and (3.26). As far as estimate (3.29) is concerned, this can now be recovered by using $(\mathrm{F})_{1}$. Indeed, multiplying (3.27) by $\Delta \varphi_{n}$ in $H$ we get

$$
\begin{aligned}
\left\|\Delta \varphi_{n}\right\|^{2} & =-\left(\mu_{n}, \Delta \varphi_{n}\right)+\left(P_{n} F^{\prime}\left(\varphi_{n}\right), \Delta \varphi_{n}\right) \\
& =-\left(\mu_{n}, \Delta \varphi_{n}\right)-\int_{\Omega} F^{\prime \prime}\left(\varphi_{n}\right)\left|\nabla \varphi_{n}\right|^{2},
\end{aligned}
$$

which yields

$$
\left\|\Delta \varphi_{n}\right\|^{2} \leq\left\|\mu_{n}\right\|^{2}+2 \lambda_{1}\left\|\nabla \varphi_{n}\right\|^{2}
$$

Estimate (3.29) then follows from this last inequality by using (3.26), the first of (3.22) and elliptic regularity.

Next, in order to get the control of the sequences of time derivatives $\varphi_{n}^{\prime}, \psi_{n}^{\prime}$ in the space $L^{r}\left(0, T ; D\left(A^{-1}\right)\right)$, for some $r>1$, and in order to pass to the limit in the approximate problem (3.17)-(3.20) we can still argue as in Step I of the proof of Theorem 1, with the difference that now we can only rely in the control given by (3.29), together with the following embedding

$$
L^{\infty}(0, T ; V) \cap L^{2}\left(0, T ; H^{2}(\Omega)\right) \hookrightarrow L^{4 \eta /(\eta-6)}\left(0, T ; L^{\eta}(\Omega)\right), \quad \text { for } 6 \leq \eta \leq \infty .
$$

Indeed, by using (3.45) with $\eta=42 / 5$ we can easily see that, since $q \in[1,7)$, estimates (3.35) and the strong convergence (3.37) still hold.

As far as the energy inequality (3.11) is concerned, let us observe that the sequence of $\varphi_{n}$ is now bounded in $L^{10}(Q)$ (cf. (3.29) and (3.45) with $\eta=10$ ). Hence, on account of (3.3) and of pointwise convergence we have $\sqrt{p\left(\varphi_{n}\right)} \rightarrow \sqrt{p(\varphi)}$ strongly in $L^{\delta}(Q)$, for every $\delta<20 / q$. In particular we have $\sqrt{p\left(\varphi_{n}\right)} \rightarrow \sqrt{p(\varphi)}$ strongly in $L^{5 / 2}(Q)$, which implies that

$$
\sqrt{p\left(\varphi_{n}\right)}\left(\mu_{n}-\psi_{n}\right) \rightarrow \sqrt{p(\varphi)}(\mu-\psi), \quad \text { in } L^{10 / 9}(Q)
$$

Due to $(3.23)_{3}$, this weak convergence also holds in $L^{2}(Q)$ and still yields (3.38) and then (3.11) as well.

The next result is concerned with uniqueness of weak solutions and their continuous dependence with respect to the initial data. In order to prove such a result assumption (F) still suffices, but we need to strengthen (P) as follows 
(P1) Let $p \in C_{l o c}^{0,1}(\mathbb{R})$ be such that $p \geq 0$ and

$$
\left|p^{\prime}(s)\right| \leq c_{5}\left(1+|s|^{q-1}\right)
$$

for almost any $s \in \mathbb{R}$, with $1 \leq q \leq 4$.

Then we have

Theorem 2. Assume that (F) and (P1) are satisfied. Let $\varphi_{0} \in V$ and $\psi_{0} \in H$. Then, for every $T>0$ the weak solution to Problem (1.1)-(1.5) on $[0, T]$ given by Theorem 1 is unique. Moreover, let $\left[\varphi_{0 i}, \psi_{0 i}\right] \in V \times H$, be two initial data and $\left[\varphi_{i}, \psi_{i}\right], i=1,2$ be the corresponding weak solutions. Then, the following continuous dependence estimate holds

$$
\begin{aligned}
& \left\|\varphi_{2}(t)-\varphi_{1}(t)\right\|_{V^{\prime}}+\left\|\psi_{2}(t)-\psi_{1}(t)\right\|_{V^{\prime}}+\left\|\varphi_{2}-\varphi_{1}\right\|_{L^{2}(0, t ; V)}+\left\|\psi_{2}-\psi_{1}\right\|_{L^{2}(0, t ; H)} \\
& \leq \Lambda(t)\left(\left\|\varphi_{02}-\varphi_{01}\right\|_{V^{\prime}}+\left\|\psi_{02}-\psi_{01}\right\|_{V^{\prime}}\right), \quad \forall t \in[0, T],
\end{aligned}
$$

where $\Lambda$ is a continuous positive function which depends on the norms of the initial data and on $F, p, \Omega$ and $T$.

Remark 2. Notice that the restriction $1 \leq q \leq 4$ on the growth of $p$ which is needed to establish the uniqueness is exactly the same condition which ensures the validity of the energy identity (3.40) which is proven in Theorem 1.

Proof. Let us rewrite the chemical potential $\mu$ and (3.7)-(3.8) in the following form

$$
\begin{aligned}
& \left\langle\varphi_{t}, \chi\right\rangle+(\nabla \mu, \nabla \chi)+(\mu, \chi)=(p(\varphi) \psi-(p(\varphi)-1) \mu, \chi), \\
& \mu=A \varphi+G^{\prime}(\varphi) \\
& \left\langle\psi_{t}, \xi\right\rangle+(\nabla \psi, \nabla \xi)+(\psi, \xi)=-((p(\varphi)-1) \psi+p(\varphi) \mu, \xi),
\end{aligned}
$$

for all $\chi, \xi \in V$, where $G(s):=F(s)-\frac{1}{2} s^{2}$.

We now write system (3.46)-(3.48) for two weak solutions $\left[\varphi_{i}, \psi_{i}\right], i=1,2$, and take the difference of each equation. Setting $\varphi:=\varphi_{2}-\varphi_{1}, \psi:=\psi_{2}-\psi_{1}$ and $\mu:=\mu_{2}-\mu_{1}$, we have

$$
\begin{aligned}
& \left\langle\varphi_{t}, \chi\right\rangle+(\nabla \mu, \nabla \chi)+(\mu, \chi) \\
& =\left(\left(p\left(\varphi_{2}\right)-p\left(\varphi_{1}\right)\right)\left(\psi_{2}-\mu_{2}\right)+p\left(\varphi_{1}\right) \psi-\left(p\left(\varphi_{1}\right)-1\right) \mu, \chi\right) \\
& \mu=A \varphi+G^{\prime}\left(\varphi_{2}\right)-G^{\prime}\left(\varphi_{1}\right) \\
& \left\langle\psi_{t}, \xi\right\rangle+(\nabla \psi, \nabla \xi)+(\psi, \xi) \\
& =-\left(\left(p\left(\varphi_{2}\right)-p\left(\varphi_{1}\right)\right)\left(\psi_{2}-\mu_{2}\right)-\left(p\left(\varphi_{1}\right)-1\right) \psi+p\left(\varphi_{1}\right) \mu, \xi\right),
\end{aligned}
$$

for all $\chi, \xi \in V$. Let us take $\chi=A^{-1} \varphi$ in (3.49) and $\xi=A^{-1} \psi$ in (3.51) and sum the resulting identities. Taking also (3.50) into account, we get

$$
\frac{1}{2} \frac{d}{d t}\|\varphi\|_{V^{\prime}}^{2}+\|\varphi\|_{V}^{2}+\left(G^{\prime}\left(\varphi_{2}\right)-G^{\prime}\left(\varphi_{1}\right), \varphi\right)+\frac{1}{2} \frac{d}{d t}\|\psi\|_{V^{\prime}}^{2}+\|\psi\|^{2}
$$




$$
\begin{aligned}
& =\left(\left(p\left(\varphi_{2}\right)-p\left(\varphi_{1}\right)\right)\left(\psi_{2}-\mu_{2}\right)+p\left(\varphi_{1}\right) \psi-\left(p\left(\varphi_{1}\right)-1\right) \mu, A^{-1} \varphi\right) \\
& +\left(-\left(p\left(\varphi_{2}\right)-p\left(\varphi_{1}\right)\right)\left(\psi_{2}-\mu_{2}\right)-\left(p\left(\varphi_{1}\right)-1\right) \psi+p\left(\varphi_{1}\right) \mu, A^{-1} \psi\right) .
\end{aligned}
$$

We now need to estimate the terms on the right hand side. Observe first that

$$
\left(p\left(\varphi_{1}\right) \psi-\left(p\left(\varphi_{1}\right)-1\right) \mu, A^{-1} \varphi\right) \leq\left(\left\|p\left(\varphi_{1}\right)(\psi-\mu)\right\|_{V^{\prime}}+\|\mu\|_{V^{\prime}}\right)\|\varphi\|_{V^{\prime}}
$$

We have to estimate in $V^{\prime}$ the term $p\left(\varphi_{1}\right)(\psi-\mu)$. Let us first estimate $p\left(\varphi_{1}\right) \chi$ in $V$. By using assumption (P1) we get

$$
\left\|p\left(\varphi_{1}\right) \nabla \chi\right\| \leq c\left(1+\left\|\varphi_{1}\right\|_{L^{\infty}(\Omega)}^{q}\right)\|\nabla \chi\| .
$$

Moreover, we have

$$
\left\|p^{\prime}\left(\varphi_{1}\right) \nabla \varphi_{1} \chi\right\| \leq\left\|p^{\prime}\left(\varphi_{1}\right) \nabla \varphi_{1}\right\|_{L^{3}(\Omega)}\|\chi\|_{L^{6}(\Omega)} \leq\left\|p^{\prime}\left(\varphi_{1}\right) \nabla \varphi_{1}\right\|_{L^{3}(\Omega)}\|\chi\|_{V}
$$

However, $\nabla \varphi_{1} \in L^{\infty}(0, T ; H) \cap L^{2}\left(0, T ; H^{2}(\Omega)\right) \hookrightarrow L^{8}\left(0, T ; L^{3}(\Omega)\right)$. On the other hand, $\varphi_{1} \in L^{\infty}(0, T ; V) \cap L^{2}\left(0, T ; H^{3}(\Omega)\right) \hookrightarrow L^{8}\left(0, T ; L^{\infty}(\Omega)\right)$ (cf. (3.33) with $\theta=\infty$ ). Thus, thanks to assumption (P1), we also have $p^{\prime}\left(\varphi_{1}\right) \in L^{8 /(q-1)}\left(0, T ; L^{\infty}(\Omega)\right)$. Hence, we find

$$
p^{\prime}\left(\varphi_{1}\right) \nabla \varphi_{1} \in L^{8 / q}\left(0, T ; L^{3}(\Omega)\right) .
$$

Moreover, observe that

$$
\left\|p\left(\varphi_{1}\right) \chi\right\| \leq c\left\|p\left(\varphi_{1}\right)\right\|_{L^{3}(\Omega)}\|\chi\|_{V}
$$

and

$$
\left\|p\left(\varphi_{1}\right)\right\|_{L^{3}(\Omega)} \leq c\left(1+\left\|\varphi_{1}\right\|_{L^{3 q}(\Omega)}^{q}\right) .
$$

Observing that $\varphi_{1} \in L^{\infty}(0, T ; V) \cap L^{2}\left(0, T ; H^{3}(\Omega)\right) \hookrightarrow L^{8 q /(q-2)}\left(0, T ; L^{3 q}(\Omega)\right)$ (cf. (3.33) $)$, then we have

$$
p\left(\varphi_{1}\right) \in L^{8 /(q-2)}\left(0, T ; L^{3}(\Omega)\right) .
$$

By collecting (3.54)-(3.59) we get

$$
\left\|p\left(\varphi_{1}\right) \chi\right\|_{V} \leq \alpha_{1}(t)\|\chi\|_{V}
$$

where the function $\alpha_{1}$ is given by

$$
\alpha_{1}(t):=c\left(\left\|p\left(\varphi_{1}(t)\right)\right\|_{L^{3}(\Omega)}+\left\|\varphi_{1}(t)\right\|_{L^{\infty}(\Omega)}^{q}+\left\|p^{\prime}\left(\varphi_{1}(t)\right) \nabla \varphi_{1}(t)\right\|_{L^{3}(\Omega)}+1\right),
$$

and, since $q \leq 4$, we have $\alpha_{1} \in L^{2}(0, T)$. Therefore, we obtain

$$
\left|\left\langle p\left(\varphi_{1}\right)(\psi-\mu), \chi\right\rangle\right| \leq\left|\left((\psi-\mu), p\left(\varphi_{1}\right) \chi\right)\right| \leq \alpha_{1}(t)\|\psi-\mu\|_{V^{\prime}}\|\chi\|_{V}
$$


which yields

$$
\left\|p\left(\varphi_{1}\right)(\psi-\mu)\right\|_{V^{\prime}} \leq \alpha_{1}(t)\|\psi-\mu\|_{V^{\prime}}
$$

By combining (3.53) with (3.60) we deduce

$$
\left(p\left(\varphi_{1}\right) \psi-\left(p\left(\varphi_{1}\right)-1\right) \mu, A^{-1} \varphi\right) \leq \alpha_{1}(t)\left(\|\psi\|_{V^{\prime}}+\|\mu\|_{V^{\prime}}\right)\|\varphi\|_{V^{\prime}}
$$

For the estimate of $\mu$ in $V^{\prime}$, by means of assumption (F) and using the continuous embedding $L^{6 / 5}(\Omega) \hookrightarrow V^{\prime}$, it is easy to see that

$$
\begin{aligned}
\|\mu\|_{V^{\prime}} & \leq\|\varphi\|_{V}+\left\|G^{\prime}\left(\varphi_{2}\right)-G^{\prime}\left(\varphi_{1}\right)\right\|_{V^{\prime}} \\
& \leq\|\varphi\|_{V}+c\left(1+\left\|\varphi_{1}\right\|_{L^{3(\rho-2) / 2}(\Omega)}^{\rho-2}+\left\|\varphi_{2}\right\|_{L^{3(\rho-2) / 2}(\Omega)}^{\rho-2}\right)\|\varphi\|_{L^{6}(\Omega)} \\
& \leq c\left(1+\left\|\varphi_{1}\right\|_{V}^{\rho-2}+\left\|\varphi_{2}\right\|_{V}^{\rho-2}\right)\|\varphi\|_{V} \leq \Gamma\|\varphi\|_{V},
\end{aligned}
$$

since $3(\rho-2) / 2 \leq 6$, being $\rho \leq 17 / 3$. In the last inequality we have used (3.4) $)_{1}$. In (3.62) and also in the estimates below, $\Gamma$ denotes a positive constant that depends on the norms of the initial data of the two solutions, i.e., $\Gamma=\Gamma\left(\left\|\varphi_{01}\right\|_{V},\left\|\varphi_{02}\right\|_{V},\left\|\psi_{01}\right\|,\left\|\psi_{02}\right\|\right)$ (of course, $\Gamma$ depends also on $F$ and $\Omega$ ). From (3.61) and (3.62) we get

$$
\begin{aligned}
\left|\left(p\left(\varphi_{1}\right) \psi-\left(p\left(\varphi_{1}\right)-1\right) \mu, A^{-1} \varphi\right)\right| & \leq \alpha_{1}(t) \Gamma\left(\|\psi\|_{V^{\prime}}+\|\varphi\|_{V}\right)\|\varphi\|_{V^{\prime}} \\
& \leq \frac{1}{10}\|\varphi\|_{V}^{2}+\Gamma \alpha_{1}^{2}(t)\left(\|\psi\|_{V^{\prime}}^{2}+\|\varphi\|_{V^{\prime}}^{2}\right) .
\end{aligned}
$$

The next term on the right hand side of (3.52) to be estimated is the following

$$
\left|\left(\left(p\left(\varphi_{2}\right)-p\left(\varphi_{1}\right)\right)\left(\psi_{2}-\mu_{2}\right), A^{-1} \varphi\right)\right| \leq\left\|\left(p\left(\varphi_{2}\right)-p\left(\varphi_{1}\right)\right)\left(\psi_{2}-\mu_{2}\right)\right\|_{V^{\prime}}\|\varphi\|_{V^{\prime}} .
$$

Let us first control the term $\left(\left(p\left(\varphi_{2}\right)-p\left(\varphi_{1}\right)\right)\left(\psi_{2}-\mu_{2}\right)\right.$ in $V^{\prime}$. We have, for every $\chi \in V$,

$$
\begin{aligned}
\left.\mid\left\langle\left(p\left(\varphi_{1}\right)-p\left(\varphi_{2}\right)\right)\left(\psi_{2}-\mu_{2}\right), \chi\right\rangle\right) \mid & \leq\left\|p\left(\varphi_{1}\right)-p\left(\varphi_{2}\right)\right\|\left\|\psi_{2}-\mu_{2}\right\|_{L^{3}}\|\chi\|_{L^{6}} \\
& \leq c\left\|p\left(\varphi_{1}\right)-p\left(\varphi_{2}\right)\right\|\left\|\psi_{2}-\mu_{2}\right\|_{L^{3}}\|\chi\|_{V} .
\end{aligned}
$$

On the other hand, thanks to (P1), we obtain

$$
\begin{aligned}
\left\|p\left(\varphi_{2}\right)-p\left(\varphi_{1}\right)\right\| & \leq c\left(1+\left\|\varphi_{1}\right\|_{L^{\infty}(\Omega)}^{q-1}+\left\|\varphi_{2}\right\|_{L^{\infty}(\Omega)}^{q-1}\right)\|\varphi\| \\
& \leq c\left(1+\left\|\varphi_{1}\right\|_{L^{\infty}(\Omega)}^{q-1}+\left\|\varphi_{2}\right\|_{L^{\infty}(\Omega)}^{q-1}\right)\|\varphi\|_{V^{\prime}}^{1 / 2}\|\varphi\|_{V}^{1 / 2} .
\end{aligned}
$$

Moreover, by using (2.1), we get

$$
\left\|\mu_{2}\right\|_{L^{3}(\Omega)} \leq c\left\|\mu_{2}\right\|^{1 / 2}\left\|\mu_{2}\right\|_{V}^{1 / 2} \leq c\left\|\mu_{2}\right\|_{V^{\prime}}^{1 / 4}\left\|\mu_{2}\right\|_{V}^{3 / 4} \leq \Gamma\left\|\varphi_{2}\right\|_{V}^{1 / 4}\left\|\mu_{2}\right\|_{V}^{3 / 4}
$$

where we have exploited the estimate $\left\|\mu_{2}\right\|_{V^{\prime}} \leq \Gamma\left\|\varphi_{2}\right\|_{V}$, which can be deduced by arguing as in (3.62). Hence, from (3.65) $-(3.67)$ we infer

$$
\left\|\left(p\left(\varphi_{2}\right)-p\left(\varphi_{1}\right)\right)\left(\psi_{2}-\mu_{2}\right)\right\|_{V^{\prime}} \leq \alpha_{2}(t)\|\varphi\|_{V^{\prime}}^{1 / 2}\|\varphi\|_{V}^{1 / 2}
$$


where

$$
\alpha_{2}(t):=c\left(1+\left\|\varphi_{1}(t)\right\|_{L^{\infty}(\Omega)}^{q-1}+\left\|\varphi_{2}(t)\right\|_{L^{\infty}(\Omega)}^{q-1}\right)\left(\left\|\psi_{2}(t)\right\|_{L^{3}(\Omega)}+\Gamma\left\|\varphi_{2}(t)\right\|_{V}^{1 / 4}\left\|\mu_{2}(t)\right\|_{V}^{3 / 4}\right) .
$$

Observe that $\alpha_{2} \in L^{4 / 3}(0, T)$ since $q \leq 4$. Indeed, both factors in (3.68) are in $L^{8 / 3}(0, T)$, recalling that $\varphi_{1}, \varphi_{2} \in L^{\infty}(0, T ; V) \cap L^{2}\left(0, T ; H^{3}(\Omega)\right) \hookrightarrow L^{8}\left(0, T ; L^{\infty}(\Omega)\right)$ and properties (3.4)-(3.6). Hence, from (3.64) we get

$$
\begin{aligned}
\left|\left(\left(p\left(\varphi_{2}\right)-p\left(\varphi_{1}\right)\right)\left(\psi_{2}-\mu_{2}\right), A^{-1} \varphi\right)\right| & \leq \alpha_{2}(t)\|\varphi\|_{V}^{1 / 2}\|\varphi\|_{V^{\prime}}^{3 / 2} \\
& \leq \frac{1}{10}\|\varphi\|_{V}^{2}+\alpha_{2}^{4 / 3}(t)\|\varphi\|_{V^{\prime}}^{2} .
\end{aligned}
$$

We now estimate the following term (cf. the right hand side of (3.52)

$$
\begin{aligned}
\left|\left(-\left(p\left(\varphi_{2}\right)-p\left(\varphi_{1}\right)\right)\left(\psi_{2}-\mu_{2}\right), A^{-1} \psi\right)\right| & \leq\left\|\left(p\left(\varphi_{2}\right)-p\left(\varphi_{1}\right)\right)\left(\psi_{2}-\mu_{2}\right)\right\|_{V^{\prime}}\|\psi\|_{V^{\prime}} \\
& \leq \alpha_{2}(t)\|\varphi\|_{V^{\prime}}^{1 / 2}\|\varphi\|_{V}^{1 / 2}\|\psi\|_{V^{\prime}} \\
& \leq \frac{1}{10}\|\varphi\|_{V}^{2}+\alpha_{2}^{4 / 3}(t)\|\varphi\|_{V^{\prime}}^{2 / 3}\|\psi\|_{V^{\prime}}^{4 / 3} \\
& \leq \frac{1}{10}\|\varphi\|_{V}^{2}+\alpha_{2}^{4 / 3}(t)\left(\|\varphi\|_{V^{\prime}}^{2}+\|\psi\|_{V^{\prime}}^{2}\right) .
\end{aligned}
$$

We now estimate the last term on the right hand side of (3.52)

$$
\begin{aligned}
\left|\left(-\left(p\left(\varphi_{1}\right)-1\right) \psi+p\left(\varphi_{1}\right) \mu, A^{-1} \psi\right)\right| & \leq\left(\left\|p\left(\varphi_{1}\right)(\psi-\mu)\right\|_{V^{\prime}}+\|\psi\|_{V^{\prime}}\right)\|\psi\|_{V^{\prime}} \\
& \leq\left(\alpha_{1}\|\psi-\mu\|_{V^{\prime}}+\|\psi\|_{V^{\prime}}\right)\|\psi\|_{V^{\prime}} \\
& \leq \alpha_{1}\|\psi\|_{V^{\prime}}^{2}+\alpha_{1} \Gamma\|\varphi\|_{V}\|\psi\|_{V^{\prime}} \\
& \leq \frac{1}{10}\|\varphi\|_{V}^{2}+\Gamma \alpha_{1}^{2}(t)\|\psi\|_{V^{\prime}}^{2}
\end{aligned}
$$

where we have used (3.60) and (3.62).

Moreover, setting $\beta:=c_{3}+1$, we have

$$
\left(G^{\prime}\left(\varphi_{2}\right)-G^{\prime}\left(\varphi_{1}\right), \varphi\right) \geq-\beta\|\varphi\|^{2} \geq-\frac{1}{10}\|\varphi\|_{V}^{2}-c\|\varphi\|_{V^{\prime}}^{2}
$$

Finally, plugging estimates (3.63) and (3.69)-(3.72) into (3.52) yields the following differential inequality

$$
\frac{d}{d t}\left(\|\varphi\|_{V^{\prime}}^{2}+\|\psi\|_{V^{\prime}}^{2}\right)+\|\varphi\|_{V}^{2}+\|\psi\|^{2} \leq \gamma\left(\|\varphi\|_{V^{\prime}}^{2}+\|\psi\|_{V^{\prime}}^{2}\right),
$$

where

$$
\gamma:=\Gamma\left(\alpha_{1}^{2}+\alpha_{2}^{4 / 3}+1\right) \in L^{1}(0, T) .
$$

An application of Gronwall's inequality to (3.73) ends the proof. 


\section{Strong solutions and the global attractor}

Here we establish a regularity result for Problem (1.1)-(1.5) that holds under the same condition on $p$ which ensures uniqueness (cf. (P1)). This result will be used to deduce some uniform in time higher-order estimates which will be crucial in order to prove the existence of the global attractor.

Theorem 3. Suppose $(F)$ and (P1) hold. Let $\varphi_{0} \in H^{3}(\Omega)$ and $\psi_{0} \in V$. Then, for every $T>0$, the solution $[\varphi, \psi]$ to Problem (1.1) -(1.5) on $[0, T]$ given by Theorem 11 satisfies

$$
\begin{aligned}
& \varphi \in L^{\infty}\left(0, T ; H^{3}(\Omega)\right), \quad \varphi_{t} \in L^{2}(0, T ; V), \\
& \mu \in L^{\infty}(0, T ; V), \\
& \psi \in L^{\infty}(0, T ; V), \quad \psi_{t} \in L^{2}(0, T ; H) .
\end{aligned}
$$

Proof. The proof is carried out by deducing formally some higher order identities and estimates which can be justified rigorously by means of a suitable approximation procedure (see the proof of Theorem 11).

Testing (1.1) by $\mu_{t}$ in $H$ and using (1.2), we find

$$
\frac{1}{2} \frac{d}{d t}\|\nabla \mu\|^{2}+\left\|\nabla \varphi_{t}\right\|^{2}+\int_{\Omega} F^{\prime \prime}(\varphi) \varphi_{t}^{2}=\left(p(\varphi)(\psi-\mu), \mu_{t}\right)
$$

whence

$$
\frac{1}{2} \frac{d}{d t}\|\nabla \mu\|^{2}+\left\|\nabla \varphi_{t}\right\|^{2}+\int_{\Omega} F^{\prime \prime}(\varphi) \varphi_{t}^{2}+\frac{1}{2} \frac{d}{d t} \int_{\Omega} p(\varphi) \mu^{2}=\frac{1}{2} \int_{\Omega} p^{\prime}(\varphi) \varphi_{t} \mu^{2}+\left(p(\varphi) \psi, \mu_{t}\right) .
$$

Test now (1.3) by $\psi_{t}$ in $H$ to get

$$
\left\|\psi_{t}\right\|^{2}=-\frac{1}{2} \frac{d}{d t}\|\nabla \psi\|^{2}-\frac{1}{2} \frac{d}{d t} \int_{\Omega} p(\varphi) \psi^{2}+\frac{1}{2} \int_{\Omega} p^{\prime}(\varphi) \varphi_{t} \psi^{2}+\left(p(\varphi) \mu, \psi_{t}\right) .
$$

Summing (4.1) with (4.2) we obtain

$$
\begin{aligned}
& \frac{1}{2} \frac{d}{d t}\|\nabla \mu\|^{2}+\left\|\nabla \varphi_{t}\right\|^{2}+\int_{\Omega} F^{\prime \prime}(\varphi) \varphi_{t}^{2}+\frac{1}{2} \frac{d}{d t} \int_{\Omega} p(\varphi) \mu^{2} \\
& +\left\|\psi_{t}\right\|^{2}+\frac{1}{2} \frac{d}{d t}\|\nabla \psi\|^{2}+\frac{1}{2} \frac{d}{d t} \int_{\Omega} p(\varphi) \psi^{2} \\
& =\frac{1}{2} \int_{\Omega} p^{\prime}(\varphi) \varphi_{t} \mu^{2}+\frac{d}{d t} \int_{\Omega} p(\varphi) \psi \mu-\int_{\Omega} p^{\prime}(\varphi) \varphi_{t} \psi \mu+\frac{1}{2} \int_{\Omega} p^{\prime}(\varphi) \varphi_{t} \psi^{2}
\end{aligned}
$$

so that

$$
\frac{1}{2} \frac{d}{d t}\left(\|\nabla \mu\|^{2}+\|\nabla \psi\|^{2}+\int_{\Omega} p(\varphi)(\mu-\psi)^{2}\right)+\left\|\nabla \varphi_{t}\right\|^{2}+\int_{\Omega} F^{\prime \prime}(\varphi) \varphi_{t}^{2}+\left\|\psi_{t}\right\|^{2}
$$




$$
=\frac{1}{2} \int_{\Omega} p^{\prime}(\varphi) \varphi_{t}(\mu-\psi)^{2} .
$$

Observe now that

$$
\left|\frac{1}{2} \int_{\Omega} p^{\prime}(\varphi) \varphi_{t}(\mu-\psi)^{2}\right| \leq \frac{1}{2}\left\|p^{\prime}(\varphi)\right\|\left\|\varphi_{t}\right\|_{L^{6}(\Omega)}\|\mu-\psi\|_{L^{6}(\Omega)}^{2} \leq c\left\|p^{\prime}(\varphi)\right\|\left\|\varphi_{t}\right\|_{V}\|\mu-\psi\|_{V}^{2} .
$$

Moreover, we have (using (1.1) and (1.4))

$$
\begin{aligned}
\left\|\varphi_{t}\right\|_{V} & \leq\left(1+c_{\Omega}\right)\left\|\nabla \varphi_{t}\right\|+|\Omega|^{1 / 2}\left|\bar{\varphi}_{t}\right| \\
& \leq\left(1+c_{\Omega}\right)\left\|\nabla \varphi_{t}\right\|+\frac{1}{|\Omega|^{1 / 2}}\left|\int_{\Omega} p(\varphi)(\mu-\psi)\right| \\
& \leq\left(1+c_{\Omega}\right)\left\|\nabla \varphi_{t}\right\|+\frac{1}{|\Omega|^{1 / 2}}\|p(\varphi)\|_{L^{6 / 5}(\Omega)}\|\mu-\psi\|_{L^{6}(\Omega)},
\end{aligned}
$$

where $c_{\Omega}$ is the constant appearing in the Poincaré-Wirtinger inequality. Hence, by combining (4.4) with (4.5), we get

$$
\begin{aligned}
& \left|\frac{1}{2} \int_{\Omega} p^{\prime}(\varphi) \varphi_{t}(\mu-\psi)^{2}\right| \leq c\left\|p^{\prime}(\varphi)\right\|\left(\left\|\nabla \varphi_{t}\right\|+\|p(\varphi)\|_{L^{6 / 5}(\Omega)}\|\mu-\psi\|_{V}\right)\left(\|\mu\|_{V}^{2}+\|\psi\|_{V}^{2}\right) \\
& \leq \frac{1}{2}\left\|\nabla \varphi_{t}\right\|^{2}+c\left(1+\left\|p^{\prime}(\varphi)\right\|^{2}\right)\left(\|\mu\|_{V}^{4}+\|\psi\|_{V}^{4}\right)+c\left\|p^{\prime}(\varphi)\right\|^{4}\|p(\varphi)\|_{L^{6 / 5}(\Omega)}^{4} .
\end{aligned}
$$

Thanks to (P1) and to (3.4 $)_{1}$ we can see that $p^{\prime}(\varphi)$ is controlled in $L^{\infty}(0, T ; H)$. Moreover, we know that $\varphi$ is bounded in $L^{18}\left(0, T ; L^{54 / 5}(\Omega)\right.$ ) (cf. (3.34)) and $\varphi$ is also bounded in $L^{4 q}\left(0, T ; L^{6 q / 5}(\Omega)\right)$ since $q \leq 4$, Thanks to this bound, assumption (P1) entails that $p(\varphi)$ is controlled in $L^{4}\left(0, T ; L^{6 / 5}(\Omega)\right)$. Thus we have

$$
\left\|p^{\prime}(\varphi)\right\|_{L^{\infty}(0, T ; H)} \leq \Gamma, \quad\|p(\varphi)\|_{L^{4}\left(0, T ; L^{6 / 5}(\Omega)\right)} \leq \Gamma,
$$

where henceforth $\Gamma=\Gamma\left(\left\|\varphi_{0}\right\|_{V},\left\|\psi_{0}\right\|\right)$ will denote a positive constant that depends on the norms of the initial data (and on $F, p, \Omega$ ). Furthermore, we have

$$
\begin{aligned}
& \|\mu\|_{V} \leq\left(1+c_{\Omega}\right)\|\nabla \mu\|+|\Omega|^{1 / 2}|\bar{\mu}| \leq\left(1+c_{\Omega}\right)\|\nabla \mu\|+\Gamma, \\
& \|\psi\|_{V} \leq\|\nabla \psi\|+\Gamma .
\end{aligned}
$$

Plugging estimate (4.6) into (4.3) and using (4.7), (4.8), (4.9) and (3.2), we get

$$
\begin{aligned}
& \frac{1}{2} \frac{d}{d t}\left(\|\nabla \mu\|^{2}+\|\nabla \psi\|^{2}+\int_{\Omega} p(\varphi)(\mu-\psi)^{2}\right)+\frac{1}{2}\left\|\nabla \varphi_{t}\right\|^{2}+\left\|\psi_{t}\right\|^{2} \leq c_{3}\left\|\varphi_{t}\right\|^{2} \\
& +\Gamma\left(\|\mu\|_{V}^{2}\|\nabla \mu\|^{2}+\|\psi\|_{V}^{2}\|\nabla \psi\|^{2}\right)+\Gamma\left(\|\mu\|_{V}^{2}+\|\psi\|_{V}^{2}+\|p(\varphi)\|_{L^{6 / 5}(\Omega)}^{4}\right) .
\end{aligned}
$$

We now need an estimate for the $L^{2}$-norm of $\varphi_{t}$ in (4.10). This can be obtained by testing (1.1) by $\varphi_{t}$ in $H$, integrating by parts in $\Omega$ and using (1.2). This yields

$$
\left\|\varphi_{t}\right\|^{2}=\left(\mu, \Delta \varphi_{t}\right)+\left(p(\varphi)(\psi-\mu), \varphi_{t}\right)
$$




$$
=-\frac{1}{2} \frac{d}{d t}\|\Delta \varphi\|^{2}-\int_{\Omega} F^{\prime \prime}(\varphi) \nabla \varphi \cdot \nabla \varphi_{t}+\left(p(\varphi)(\psi-\mu), \varphi_{t}\right) .
$$

Hence, we have

$$
\begin{aligned}
& \frac{1}{2} \frac{d}{d t}\|\Delta \varphi\|^{2}+\frac{1}{2}\left\|\varphi_{t}\right\|^{2} \leq\left|\int_{\Omega} F^{\prime \prime}(\varphi) \nabla \varphi \cdot \nabla \varphi_{t}\right|+\frac{1}{2}\|p(\varphi)\|_{L^{3}(\Omega)}^{2}\|\mu-\psi\|_{L^{6}(\Omega)}^{2} \\
& \leq\left\|F^{\prime \prime}(\varphi)\right\|_{L^{7 / 2}(\Omega)}\|\nabla \varphi\|_{L^{14 / 3}(\Omega)}\left\|\nabla \varphi_{t}\right\|+c\|p(\varphi)\|_{L^{3}(\Omega)}^{2}\left(\|\mu\|_{V}^{2}+\|\psi\|_{V}^{2}\right) \\
& \leq \frac{1}{8 c_{3}}\left\|\nabla \varphi_{t}\right\|^{2}+c\left\|F^{\prime \prime}(\varphi)\right\|_{L^{7 / 2}(\Omega)}^{2}\|\nabla \varphi\|_{L^{14 / 3}(\Omega)}^{2}+c\|p(\varphi)\|_{L^{3}(\Omega)}^{2}\left(\|\mu\|_{V}^{2}+\|\psi\|_{V}^{2}\right) .
\end{aligned}
$$

Recalling that $\varphi$ is bounded in $L^{14}(Q)$ (cf (3.30) and (3.33) with $\theta=14$ ), (F) implies that $F^{\prime \prime}(\varphi)$ is bounded in $L^{7 / 2}(Q)$ (note that $\rho<17 / 3<6$ ). Moreover, $\nabla \varphi$ is bounded in $L^{14 / 3}(Q)$ (cf. (3.30) and (3.44) with $s=2$ ). Therefore the second term on the right hand side of the last inequality in (4.11) is bounded in $L^{1}(0, T)$.

Furthermore, $\varphi$ is also bounded in $L^{8}\left(0, T ; L^{\infty}(\Omega)\right.$ ) (cf. (3.30) and (3.33) with $\theta=\infty$ ) and, being $q \leq 4,(\mathrm{P} 1)$ implies that $p(\varphi)$ is bounded in $L^{2}\left(0, T ; L^{3}(\Omega)\right)$.

By combining (4.10) with (4.11), also on account of (4.8) and (4.9), we obtain the following differential inequality

$$
\begin{aligned}
& \frac{1}{2} \frac{d}{d t}\left(\|\nabla \mu\|^{2}+\|\nabla \psi\|^{2}+2 c_{3}\|\Delta \varphi\|^{2}+\int_{\Omega} p(\varphi)(\mu-\psi)^{2}\right)+\frac{1}{4}\left\|\nabla \varphi_{t}\right\|^{2}+\left\|\psi_{t}\right\|^{2} \\
& \leq \sigma_{1}\left(\|\nabla \mu\|^{2}+\|\nabla \psi\|^{2}\right)+\sigma_{2}
\end{aligned}
$$

where

$$
\sigma_{1}:=c\|p(\varphi)\|_{L^{3}(\Omega)}^{2}, \quad \sigma_{2}:=c\left\|F^{\prime \prime}(\varphi)\right\|_{L^{7 / 2}(\Omega)}^{2}\|\nabla \varphi\|_{L^{14 / 3}(\Omega)}^{2}+\Gamma\|p(\varphi)\|_{L^{3}(\Omega)}^{2} .
$$

Notice that

$$
\left\|\sigma_{1}\right\|_{L^{1}(0, T)} \leq \Gamma, \quad\left\|\sigma_{2}\right\|_{L^{1}(0, T)} \leq \Gamma .
$$

Using Gronwall's lemma and recalling the assumptions on the initial data (in particular, $\varphi_{0} \in H^{3}(\Omega)$ implies that $\mu(0) \in V$ ) from (4.12) we get that $\nabla \mu$ and $\Delta \varphi$ belong to $L^{\infty}(0, T ; H), \psi \in L^{\infty}(0, T ; V), \nabla \varphi_{t}$ and $\psi_{t}$ belong to $L^{2}(0, T ; H)$. Also, thanks to (F), we have that $F^{\prime}(\varphi) \in L^{\infty}(0, T ; H)$. Therefore $\mu \in L^{\infty}(0, T ; H)$ so that

$$
\mu \in L^{\infty}(0, T ; V) .
$$

Moreover, due to elliptic regularity result for the homogeneous Neumann problem, we deduce $\varphi \in L^{\infty}\left(0, T ; H^{2}(\Omega)\right)$. From this property and (4.14) we infer we have also

$$
\varphi \in L^{\infty}\left(0, T ; H^{3}(\Omega)\right) .
$$

Indeed, since $\varphi \in L^{\infty}\left(0, T ; H^{2}(\Omega)\right)$, we have $F^{\prime}(\varphi) \in L^{\infty}(0, T ; V)$. From (4.14) we then get $\Delta \varphi \in L^{\infty}(0, T ; V)$ and (4.15) follows by standard elliptic regularity.

Finally, as far as $\varphi_{t}$ is concerned, by integrating (4.11) in time between 0 and $t$ we get $\varphi_{t} \in L^{2}(0, T ; H)$ and this bound together with the bound for $\nabla \varphi_{t}$ deduced above imply $\varphi_{t} \in L^{2}(0, T ; V)$. 
We now show that (1.1) -(1.4) define a dynamical system on a suitable phase space. Let $M>0$ be given. Set

$$
\mathcal{W}_{M}:=\{w=[\varphi, \psi] \in V \times H: \mathcal{E}(w) \leq M\} .
$$

and endow $\mathcal{W}_{M}$ with the metric

$$
\mathbf{d}_{\mathcal{W}_{M}}\left(w_{2}, w_{1}\right):=\left\|\varphi_{2}-\varphi_{1}\right\|_{V}+\left\|\psi_{2}-\psi_{1}\right\|, \quad \forall w_{i}:=\left[\varphi_{i}, \psi_{i}\right] \in \mathcal{W}_{M}, \quad i=1,2,
$$

so that it is a complete metric space. As a consequence of Theorem 1 and Theorem 2 , assuming that (F) and (P1) are satisfied, we can define a semigroup $\left\{S_{M}(t)\right\}_{t \geq 0}$ of closed operators on $\mathcal{W}_{M}$ (cf. [25]) by setting

$$
[\varphi(t), \psi(t)]=S_{M}(t)\left[\varphi_{0}, \psi_{0}\right], \quad \forall t \geq 0,
$$

where $[\varphi, \psi]$ is the unique (weak) solution to Problem (1.1)-(1.5).

Notice that we have the total mass constraint

$$
|\overline{\varphi(t)}+\overline{\psi(t)}|=\left|\bar{\varphi}_{0}+\bar{\psi}_{0}\right| \leq Q(M), \quad \forall t \geq 0
$$

where henceforth by $Q=Q(M)$ we denote a nonnegative continuous monotone increasing function of $M$ (which may also depend on $F, p$ and $\Omega$ ). Such function may change even within the same line.

Theorem 4. Let (F) and (P1) be satisfied. Then the dynamical system $\left(\mathcal{W}_{M},\left\{S_{M}(t)\right\}_{t \geq 0}\right)$ possesses the global attractor.

Proof. Let us write (4.12) in the form

$$
\frac{d \Phi}{d t}+\frac{1}{4}\left\|\nabla \varphi_{t}\right\|^{2}+\left\|\psi_{t}\right\|^{2} \leq \sigma_{1} \Phi+\sigma_{2}
$$

where

$$
\Phi:=\frac{1}{2}\|\nabla \mu\|^{2}+\frac{1}{2}\|\nabla \psi\|^{2}+c_{3}\|\Delta \varphi\|^{2}+\frac{1}{2} \int_{\Omega} p(\varphi)(\mu-\psi)^{2} .
$$

and $\sigma_{1}$ and $\sigma_{2}$ are defined as in (4.13). Notice that, since $\Gamma=\Gamma\left(\left\|\varphi_{0}\right\|_{V},\left\|\psi_{0}\right\|\right)$ and since $\left[\varphi_{0}, \psi_{0}\right] \in \mathcal{W}_{M}$, then the constant $\Gamma$ that bounds the $L^{1}$-norm of $\sigma_{2}$ will depend only on $M$.

Integrating the energy identity (3.40) between $t$ and $t+1$, we get, for all $t \geq 0$.

$$
\int_{t}^{t+1}\|\nabla \mu\|^{2} d \tau \leq M, \quad \int_{t}^{t+1}\|\nabla \psi\|^{2} d \tau \leq M, \quad \int_{t}^{t+1} \int_{\Omega} p(\varphi)(\mu-\psi)^{2} \leq M .
$$

Recalling that $q \leq 4$, we deduce from (P1) that

$$
\int_{t}^{t+1} \sigma_{1}(\tau) d \tau \leq c\left(1+\|\varphi\|_{L^{2 q}\left(t, t+1 ; L^{3 q}(\Omega)\right)}^{2 q}\right)
$$




$$
\leq c\left(1+\|\varphi\|_{L^{\infty}(t, t+1 ; V)}^{2 q}+\|\varphi\|_{L^{2}\left(t, t+1 ; H^{3}(\Omega)\right)}^{2 q}\right) \leq Q(M) .
$$

Moreover, on account of (F) and (P1), we obtain

$$
\begin{aligned}
& \int_{t}^{t+1} \sigma_{2}(\tau) d \tau \leq c\left\|F^{\prime \prime}(\varphi)\right\|_{L^{7 / 2}\left(t, t+1 ; L^{7 / 2}(\Omega)\right)}^{2}\|\nabla \varphi\|_{L^{14 / 3}\left(t, t+1 ; L^{14 / 3}(\Omega)\right)}^{2}+Q(M) \\
& \leq c\left(1+\|\varphi\|_{L^{7(\rho-2) / 2}\left(t, t+1 ; L^{7(\rho-2) / 2}(\Omega)\right.}^{2(\rho-2)}\right)\|\nabla \varphi\|_{L^{14 / 3}\left(t, t+1 ; L^{14 / 3}(\Omega)\right)}^{2}+Q(M) \\
& \leq c\left(1+\|\varphi\|_{L^{\infty}(t, t+1 ; V)}^{2(\rho-2)}+\|\varphi\|_{L^{2}\left(t, t+1 ; H^{3}(\Omega)\right)}^{2(\rho)}\right)\left(\|\nabla \varphi\|_{L^{\infty}(t, t+1 ; H)}^{2}+\|\nabla \varphi\|_{L^{2}\left(t, t+1 ; H^{2}(\Omega)\right)}^{2}\right) \\
& +Q(M) \leq c\left(1+\|\varphi\|_{L^{\infty}(t, t+1 ; V)}^{2(\rho-1)}+\|\varphi\|_{L^{2}\left(t, t+1 ; H^{3}(\Omega)\right)}^{2(\rho-1)}\right)+Q(M) \leq Q(M) .
\end{aligned}
$$

In (4.18) and (4.19) we have used the fact that the $L^{2}\left(t, t+1 ; H^{3}(\Omega)\right)-$ norm of $\varphi$ can be controlled, uniformly in time, in terms of $\left\|\varphi_{0}\right\|_{V},\left\|\psi_{0}\right\|$ and hence of $M$, when $4<\rho<17 / 3$. Indeed, we can use the iteration argument outlined in the proof of Theorem 1 (cf. Step II; if $\rho=4$ no iteration is needed).

Therefore, we have (see (4.17))

$$
\int_{t}^{t+1} \Phi(\tau) d \tau \leq \frac{3 M}{2}+c_{3} \int_{t}^{t+1}\|\Delta \varphi(\tau)\|^{2} d \tau \leq Q(M) .
$$

Thanks to (4.18)-(4.20) we can now apply the uniform Gronwall's lemma to (4.16) and obtain

$$
\Phi(t) \leq Q(M), \quad \forall t \geq 1
$$

On the other hand, the definition of the phase space $\mathcal{W}_{M}$ and (3.2) yield

$$
\|\varphi(t)\|_{L^{\rho}(\Omega)} \leq Q(M), \quad\|\psi(t)\| \leq Q(M), \quad \forall t \geq 0 .
$$

Hence, we deduce from (4.21) and (4.22) that

$$
\|\varphi(t)\|_{H^{2}(\Omega)} \leq Q(M), \quad \forall t \geq 1
$$

Moreover, (4.21) and (4.23) entail

$$
\|\mu(t)\|_{V} \leq Q(M), \quad \forall t \geq 1 .
$$

Also, using (4.23) once more, we have

$$
\left\|\nabla F^{\prime}(\varphi(t))\right\| \leq\left\|F^{\prime \prime}(\varphi(t)) \nabla \varphi(t)\right\| \leq Q(M), \quad \forall t \geq 1 .
$$

The last two bounds, (1.2) and elliptic regularity imply

$$
\|\varphi(t)\|_{H^{3}(\Omega)} \leq Q(M), \quad \forall t \geq 1
$$


Finally, from (4.21) and (4.22) 2 , we get

$$
\|\psi(t)\|_{V} \leq Q(M), \quad \forall t \geq 1 .
$$

Thanks to (4.24) and (4.25), we have thus proven that there exists $\Lambda=\Lambda(M)>0$ such that

$$
\mathcal{B}_{M}:=\left\{w:=[\varphi, \psi] \in H^{3}(\Omega) \times H^{1}(\Omega):\|\varphi\|_{H^{3}(\Omega)} \leq \Lambda,\|\psi\|_{H^{1}(\Omega)} \leq \Lambda, \quad \mathcal{E}(w) \leq M\right\}
$$

is an absorbing set for the semigroup $\left\{S_{M}(t)\right\}_{t \geq 0}$ in $\mathcal{W}_{M}$. Since $\mathcal{B}_{M}$ is also compact in $\mathcal{W}_{M}$, the conclusion follows from [25, Thm. 2].

Acknowledgment. The authors are members of the Gruppo Nazionale per l'Analisi Matematica, la Probabilità e le loro Applicazioni (GNAMPA) of the Istituto Nazionale di Alta Matematica (INdAM).

\section{References}

[1] S. Agmon, Lectures on elliptic boundary value problems, Revised edition of the 1965 original. AMS Chelsea Publishing, Providence, RI, 2010.

[2] R.P. Araujo, D.L.S. McElwain, A History of the Study of Solid Tumour Growth: The Contribution of Mathematical Modelling, Bull. Math. Biol. (2004) 66, 1039-1091.

[3] O.V. Besov, V.P. Il'in, S.M. Nikol'skii, Integral representations of functions and embedding theorems. Vol. II, Scripta Series in Mathematics. Edited by M.H. Taibleson. V. H. Winston \& Sons, Washington, D.C.; Halsted Press [John Wiley \& Sons], New York-Toronto, Ont.-London, 1979.

[4] S. Bosia, M. Conti, M. Grasselli, On the Cahn-Hilliard-Brinkman system, arXiv: 1402.6195 (math.AP).

[5] C. Chatelain, T. Balois, P. Ciarletta, M. Ben Amar, Emergence of microstructural patterns in skin cancer: a phase separation analysis in a binary mixture, New J. Phys. 13 (2011), 115013 (21 pp.).

[6] P. Colli, P. Krejčí, E. Rocca, J. Sprekels, Nonlinear evolution inclusions arising from phase change models, Czechoslovak Math. J. 57 (2007), 1067-1098.

[7] P. Colli, G. Gilardi, D. Hilhorst, On a Cahn-Hilliard type phase fiels system related to tumor growth, Discrete Contin. Dyn. Syst. Ser. A, to appear.

[8] V. Cristini, X. Li, J.S. Lowengrub, S.M. Wise, Nonlinear simulations of solid tumor growth using a mixture model: invasion and branching, J. Math. Biol. 58 (2009), 723-763. 
[9] V. Cristini, J. Lowengrub, Multiscale modeling of cancer. An Integrated Experimental and Mathematical Modeling Approach, Cambridge Univ. Press, Cambridge, 2010.

[10] H.B. Frieboes, F. Jin, Y.-L. Chuang, S.M. Wise, J.S. Lowengrub, V. Cristini, Threedimensional multispecies nonlinear tumor growth-II: Tumor invasion and angiogenesis, J. Theoret. Biol. 264 (2010), 1254-1278.

[11] A. Friedman, Partial differential equations, Holt, Rinehart and Winston, New York, 1969.

[12] E. Gagliardo, Uleriori proprietà di alcune classi di funzioni in più variabili, Ricerche Mat. 8 (1959), 24-51.

[13] G. Giacomin, J.L. Lebowitz, Phase Segregation Dynamics in Particle Systems with Long Range Interactions. I. Macroscopic Limits, J. Stat. Phys. 87 (1997), 37-61.

[14] G. Giacomin, J.L. Lebowitz, Phase Segregation Dynamics in Particle Systems with Long Range Interactions. II. Interface Motion, SIAM J. Appl. Math. 58 (1998), 1707-1729.

[15] D. Gilbarg, N. Trudinger, Elliptic partial differential equations of second order, Grundlehren der Mathematischen Wissenschaften 224. Springer-Verlag, Berlin-New York, 1977.

[16] A. Hawkins-Daarud, K.G. van der Zee, J.T. Oden, Numerical simulation of a thermodynamically consistent four-species tumor growth model, Int. J. Numer. Meth. Biomed. Engng. 28 (2011), 3-24.

[17] A. Hawkins-Daarud, S. Prudhomme, K.G. van der Zee, J.T. Oden, Bayesian calibration, validation, and uncertainty quantification of diffuse interface models of tumor growth, J. Math. Biol. 67 (2013), 1457-1485.

[18] J.-L. Lions, Quelques méthodes de résolution des problémes aux limites non linéaires, Dunod, Gauthier-Villars, Paris 1969.

[19] J.S. Lowengrub, H.B. Frieboes, F. Jin, Y.-L. Chuang, X. Li, P. Macklin, S.M. Wise, V. Cristini, Nonlinear modelling of cancer: bridging the gap between cells and tumours, Nonlinearity 23 (2010), R1-R91.

[20] J. Lowengrub, E. Titi, K. Zhao, Analysis of a mixture model of tumor growth, European J. Appl. Math. 24 (2013), 1-44.

[21] J. Nečas, Direct methods in the theory of elliptic equations, Translated from the 1967 French original by G. Tronel and A. Kufner. Editorial coordination and preface by Š. 
Nečasová and a contribution by C.G. Simader. Springer Monographs in Mathematics. Springer, Heidelberg, 2012.

[22] L. Nirenberg, On elliptic partial differential equations, Ann. Scuola Norm. Sup. Pisa 13 (1959), 115-162.

[23] J.T. Oden, A. Hawkins, S. Prudhomme, General diffuse-interface theories and an approach to predictive tumor growth modeling, Math. Models Methods Appl. Sci. 20 (2010), 477-517.

[24] J.T. Oden, E.E. Prudencio, A. Hawkins-Daarud, Selection and assessment of phenomenological models of tumor growth, Math. Models Methods Appl. Sci. 23 (2013), 1309-1338.

[25] V. Pata, S. Zelik, A Result on the existence of global attractors for semigroups of closed operators, Commun. Pure Appl. Anal. 6 (2007), 481-486.

[26] X. Wang, H. Wu, Long-time behavior for the Hele-Shaw-Cahn-Hilliard system, Asymptot. Anal. 78 (2012), 217-245.

[27] X. Wang, Z. Zhang, Well-posedness of the Hele-Shaw-Cahn-Hilliard system, Ann. Inst. H. Poincaré Anal. Non Linéaire, 30 (2013), 367-384.

[28] S.M. Wise, J.S. Lowengrub, H.B. Frieboes, V. Cristini, Three-dimensional multispecies nonlinear tumor growth-I: Model and numerical method, J. Theoret. Biol. 253 (2008), 524-543.

[29] X. Wu, G.J. van Zwieten, K.G. van der Zee, Stabilized second-order convex splitting schemes for Cahn-Hilliard models with applications to diffuse-interface tumor-growth models, Int. J. Numer. Meth. Biomed. Engng. 30 (2014), 180-203. 